\title{
Statistical analysis of relativistic electron energies for cyclotron resonance with EMIC waves observed on CRRES
}

\author{
Nigel P. Meredith, ${ }^{1}$ Richard M. Thorne, ${ }^{2}$ Richard B. Horne, ${ }^{3}$ Danny Summers, ${ }^{4,5}$ \\ Brian J. Fraser, ${ }^{6}$ and Roger R. Anderson ${ }^{7}$ \\ Received 26 September 2002; revised 27 March 2003; accepted 7 April 2003; published 26 June 2003.
}

[1] Electromagnetic ion cyclotron (EMIC) waves which propagate at frequencies below the proton gyrofrequency can undergo cyclotron resonant interactions with relativistic electrons in the outer radiation belt and cause pitch-angle scattering and electron loss to the atmosphere. Typical storm-time wave amplitudes of 1-10 nT cause strong diffusion scattering which may lead to significant relativistic electron loss at energies above the minimum energy for resonance, $E_{\text {min }}$. A statistical analysis of over 800 EMIC wave events observed on the CRRES spacecraft is performed to establish whether scattering can occur at geophysically interesting energies $(\leq 2 \mathrm{MeV})$. While $E_{\min }$ is well above $2 \mathrm{MeV}$ for the majority of these events, it can fall below $2 \mathrm{MeV}$ in localized regions of high plasma density and/or low magnetic field $\left(f_{p e} / f_{c e, e q}>10\right)$ for wave frequencies just below the hydrogen or helium ion gyrofrequencies. These lower energy scattering events, which are mainly associated with resonant L-mode waves, are found within the magnetic local time range 1300 $<$ MLT $<1800$ for $L>4.5$. The average wave spectral intensity of these events $\left(4-5 \mathrm{nT}^{2} / \mathrm{Hz}\right)$ is sufficient to cause strong diffusion scattering. The spatial confinement of these events, together with the limited set of these waves that resonate with $\leq 2 \mathrm{MeV}$ electrons, suggest that these electrons are only subject to strong scattering over a small fraction of their drift orbit. Consequently, drift-averaged scattering lifetimes are expected to lie in the range of several hours to a day. EMIC wave scattering should therefore significantly affect relativistic electron dynamics during a storm. The waves that resonate with the $\sim \mathrm{MeV}$ electrons are produced by low-energy $(\sim \mathrm{keV})$ ring current protons, which are expected to be injected into the inner magnetosphere during enhanced convection events. INDEX TERMS: 2730

Magnetospheric Physics: Magnetosphere - inner; 2772 Magnetospheric Physics: Plasma waves and instabilities; 7867 Space Plasma Physics: Wave/particle interactions; 2716 Magnetospheric Physics: Energetic particles, precipitating; KEYWORDS: EMIC waves, relativistic electrons, wave/particle interaction, outer radiation belt

Citation: Meredith, N. P., R. M. Thorne, R. B. Horne, D. Summers, B. J. Fraser, and R. R. Anderson, Statistical analysis of relativistic electron energies for cyclotron resonance with EMIC waves observed on CRRES, J. Geophys. Res., 108(A6), 1250, doi:10.1029/2002JA009700, 2003.

\section{Introduction}

[2] The flux of relativistic electrons $(E>1 \mathrm{MeV})$ in the Earth's outer radiation belt $(3<L<7)$ varies substantially

\footnotetext{
${ }^{1}$ Mullard Space Science Laboratory, University College London, Holmbury St. Mary, Surrey, UK.

${ }^{2}$ Department of Atmospheric Sciences, University of California, Los Angeles, USA.

${ }^{3}$ British Antarctic Survey, Natural Environment Research Council, Cambridge, UK.

${ }^{4}$ Department of Mathematics and Statistics, Memorial University of Newfoundland, St. John's, Newfoundland, Canada.

${ }^{5}$ Also at School of Pure and Applied Physics, University of Natal, Durban, South Africa.

${ }^{6}$ Department of Physics, University of Newcastle, New South Wales, Australia.

${ }^{7}$ Department of Physics and Astronomy, University of Iowa, Iowa City, Iowa, USA.

Copyright 2003 by the American Geophysical Union. 0148-0227/03/2002JA009700
}

during geomagnetic storms. The variability is caused by an imbalance between source and loss processes, both of which are substantially enhanced during storm periods. For a typical storm the relativistic electron flux may fall by up to two or three orders of magnitude over a period of several hours during the main phase. This is generally followed by a gradual increase over a period of a few days during the storm recovery phase to peak flux levels which may exceed the prestorm values [e.g., Meredith et al., 2002b]. However, $25 \%$ of storms lead to a net decrease in relativistic flux, when compared with prestorm levels [Reeves et al., 2003]. Intense relativistic precipitation fluxes have also been observed during the storm recovery [Lorentzen et al., 2001; Millan et al., 2002], and such loss will compete with any potential injection or acceleration process. There has been considerable recent effort directed towards understanding the processes responsible for stormtime acceleration [e.g., Li et al., 1997; Elkington et al., 1999; Summers and Ma, 2000a, 2000b; Hudson et al., 2001; Li and Temerin, 2001; Mathie and Mann, 2001; Meredith et al., 2001, 
2002a, 2002b, 2003; O'Brien et al., 2001; Friedel et al., 2002; Summers et al., 2002; Horne et al., 2003; Miyoshi et al., 2003], due in part to the potentially serious hazard posed by enhanced fluxes of relativistic electrons to spacecraft subsystems via deep dielectric charging [Vampola, 1987; Baker et al., 1994, 1998; Wrenn, 1995; Wrenn and Smith, 1996]. Recent studies [Lorentzen et al., 2001; Millan et al., 2002; Summers and Thorne, 2003] have shown that losses may occur on time-scales comparable to or even shorter than those associated with the acceleration process. A quantitative knowledge of the loss processes is therefore essential for a fundamental understanding of relativistic electron dynamics during storms. The present study concentrates on one potentially important loss process, the scattering due to resonant interactions with electromagnetic ion cyclotron (hereafter referred to as EMIC) waves and uses CRRES satellite data to deduce the conditions under which this can occur.

[3] Several mechanisms contribute to the initial stormtime flux decrease, including adiabatic effects associated with the decrease of Dst [Kim and Chan, 1997], outward drift and loss via scattering at the magnetopause [ $\mathrm{Li}$ et al., 1997], and nonadiabatic effects due to precipitation into the atmosphere during resonant interactions with EMIC waves [Thorne and Kennel, 1971; Lyons and Thorne, 1972; Summers and Thorne, 2003]. Observations of relativistic electrons indicate a significant nonadiabatic electron loss during the main phase of every storm [Kim and Chan., 1997; Desorgher et al., 2000]. Indeed, a recent study by Iles et al. [2002] suggests that the electron losses are more efficient at higher energies.

[4] EMIC waves, which propagate at frequencies below the proton gyrofrequency, are able to resonate with the relativistic electron population [Lyons and Thorne, 1972; Horne and Thorne, 1998; Summers et al., 1998], causing pitch-angle scattering and precipitation loss to the atmosphere [Thorne, 1974; Thorne and Andreoli, 1980]. Theoretical models for the wave excitation indicate that the equatorial region along the high-density duskside plasmapause [Horne and Thorne, 1993, 1994; Fraser et al., 1996; Jordanova et al., 2001] is a preferred region for proton cyclotron instability. Wave growth is driven by anisotropic $\left(T_{\perp}>T_{\|}\right)$ring current $(1-100 \mathrm{keV})$ protons, which are injected into the inner magnetosphere by enhanced Earthward convection during geomagnetic storms and substorms [e.g., Cornwall et al., 1970; Kozyra et al., 1997; Jordanova et al., 2001]. The zone of most intense wave activity is expected to be spatially localized because of the decrease in resonant energy [e.g., Cornwall et al., 1970; Perraut et al., 1976] and wave guiding by strong density gradients associated with the plasmapause [Thorne and Horne, 1997].

[5] EMIC wave events observed near geostationary orbit are most common in the afternoon sector [Bossen et al., 1976; Roux et al., 1982; Fraser and McPherron, 1982]. In this sector the EMIC occurrence rate increases monotonically with $L$ in the region $3<L<9$, with rates peaking at $10 \%-20 \%$ in the region $7<L<9,1100<$ MLT $<1500$ [Anderson et al., 1992a]. While EMIC waves may be observed during nonstorm periods, they are found to be most common and most intense during geomagnetic storms [Braysy et al., 1998; Erlandson and Ukhorskiy, 2001]. Recent observations from the CRRES spacecraft indicate that although the plasmapause is a region of wave generation, it is not the only preferred region, since significant wave power is also present in the lower density trough [Fraser and Nguyen, 2001]. EMIC waves can occur in three distinct bands below the gyrofrequencies of $\mathrm{H}^{+}, \mathrm{He}^{+}$and $\mathrm{O}^{+}$, the excited band being controlled by the ion composition and anisotropy [Kozyra et al., 1984; Horne and Thorne, 1994], the level of geomagnetic activty [Braysy et al., 1998], and the location with respect to the plasmapause [Fraser and Nguyen, 2001]. Waves in the upper ("hydrogen") band between the helium and hydrogen gyrofrequencies are mainly found outside the plasmapause, while those in the ("helium") band between the oxygen and helium gyrofrequencies may be observed both inside and outside the plasmapause. EMIC waves are only rarely observed in the band below the oxygen gyrofrequency.

[6] Evidence that EMIC waves can precipitate relativistic electrons has been deduced from balloon measurements of X-ray emissions in the dusk sector. Foat et al. [1998] and Lorentzen et al. [2000] observed an intense X-ray event at 1532 UT on 20 August 1996 from a high-altitude balloon, consistent with atmospheric bremsstrahlung from precipitating, monoenergetic $\sim 1.7 \mathrm{MeV}$ electrons. The most feasible mechanism for this selective precipitation of these relativistic electrons near dusk is pitch-angle scattering caused by resonant interactions with EMIC waves. A more extensive set of similar hard X-ray events was identified recently by Millan et al. [2002]. All of the events were confined to the duskside sector, and most occurred during the recovery phase of a geomagnetic storm.

[7] Relativistic electrons with energies $\sim 1 \mathrm{MeV}$ in the outer radiation belt $(3<L<7)$ typically drift around the Earth on time-scales of the order of $10-30 \mathrm{~min}$ and are expected to spend a small fraction $(\leq 1 \%)$ of each orbit within the zone of enhanced EMIC waves. Typical stormtime EMIC wave amplitudes of 1-10 nT usually exceed the level required for strong diffusion [Thorne and Kennel, 1971; Summers and Thorne, 2003]. However, because of the limited extent of the localized region of enhanced EMIC waves, significant relativistic electron loss only occurs over many drift orbits or several hours [Summers and Thorne, 2003]. Persistent EMIC wave activity could therefore contribute to the flux drop-out associated with the storm main phase. These waves are also excited by substorm activity during the storm recovery. The associated scattering could therefore provide an important loss mechanism which competes with acceleration processes during the recovery phase.

[8] If EMIC waves are to be effective in contributing to the loss of geophysically interesting relativistic electrons, the minimum resonant energy must fall into the $\sim \mathrm{MeV}$ range. Here we use the results of a comprehensive survey of the EMIC wave events observed on CRRES to determine the associated electron minimum resonant energies and to identify the spatial regions and conditions associated with $\leq 2 \mathrm{MeV}$ electron scattering.

\section{Instrumentation}

[9] CRRES was launched on 25 July 1990 and operated in a highly elliptical geosynchronous transfer orbit with a perigee of $305 \mathrm{~km}$, an apogee of $35,768 \mathrm{~km}$, and an 
inclination of $18^{\circ}$. The orbital period was approximately 10 hours, and the initial apogee was at a magnetic local time (MLT) of 0800 MLT. The magnetic local time of apogee decreased at a rate of approximately 1.3 hours per month until the satellite failed on 11 October 1991, when its apogee was at about 1400 MLT. The satellite swept through the heart of the radiation belts, on average approximately 5 times per day, providing good coverage of this important region for almost 15 months.

[10] The three-component EMIC wave and main field data were provided by the AFGL magnetometer [Singer et al., 1992]. The three-component fluxgate data, sampled at $16 \mathrm{~Hz}$, were analyzed orbit by orbit. A total of 830 EMIC wave events were identified from 169 of the analyzed 906 orbits, covering 96 hours of the analyzed 7248 hours or $1.33 \%$ of the total observation time [Fraser and Nguyen, 2001]. Relevant parameters, including the event start and stop times in UT, the peak frequency in $\mathrm{Hz}$, the local magnetic field strength, the deduced equatorial magnetic field strength, the transverse power spectral density, and the ellipticity were then entered into a database on an event by event basis. Since the ambient magnetic field and plasma frequency are required to compute the minimum resonant energies, the number of events used was reduced to the 807 events for which these measurements were also available.

[11] The electron plasma frequency, $f_{p e}$, which is required to calculate the particle resonant energies and the number density profiles, was derived from the University of Iowa plasma wave experiment. This experiment provided measurements of electric fields from $5.6 \mathrm{~Hz}$ to $400 \mathrm{kHz}$, using a $100 \mathrm{~m}$ tip-to-tip long wire antenna, and magnetic fields from $5.6 \mathrm{~Hz}$ to $10 \mathrm{kHz}$, using a search coil magnetometer, with a dynamic range covering a factor of at least $10^{5}$ in amplitude [Anderson et al., 1992b]. Inside the plasmasphere, emissions at the upper hybrid frequency, $f_{u h r}$, are usually well defined, and the electron plasma frequency was estimated from measurements of $f_{u h r}$, using the relationship $f_{p e}^{2}=f_{u h r}^{2}-f_{c e}^{2}$. Here $f_{c e}$, the electron gyrofrequency, is determined directly from ambient magnetic field provided by the AFGL magnetometer [Singer et al., 1992]. However, the upper hybrid frequency is not as well defined beyond the plasmapause. In this region the electron plasma frequency was estimated from the lower frequency limit of the electromagnetic continuum radiation, which is taken to be a plasma wave cutoff at the plasma frequency [Gurnett and Shaw, 1973].

\section{Analysis}

\subsection{Resonance Conditions}

[12] Wave-particle interactions are most effective under gyroresonance conditions when

$$
\omega-k_{\|} v_{\|}=\frac{n \Omega_{\sigma}}{\gamma}
$$

where $\gamma=\left(1-v^{2} / c^{2}\right)^{-1 / 2}$ and $v^{2}=v_{\perp}^{2}+v_{\|}^{2}$. Here $v_{\perp}$ and $v_{\|}$ are the particle velocities perpendicular and parallel to the ambient magnetic field, $\omega=2 \pi f$ is the wave frequency (measured in rads/s), $k_{\|}$is the parallel wave number, $n$ is the harmonic number and $\Omega_{\sigma}=q_{\sigma} B_{0} / m_{\sigma}$ is the particle gyrofrequency, where $B_{0}$ is the strength of the ambient magnetic field. The particle species is denoted by $\sigma, q_{\sigma}$ is the particle charge which includes the sign and $m_{\sigma}$ is the particle mass.

[13] For electron interaction with electromagnetic Rmode and L-mode waves propagating parallel to the magnetic field, only the first resonance can occur, and the resonance condition becomes

$$
\omega-k_{\|} v_{\|}= \pm \frac{\left|\Omega_{e}\right|}{\gamma}
$$

where the upper and lower signs refer to the R-mode and Lmode, respectively. For proton interaction with electromagnetic R-mode and L-mode waves propagating parallel to the magnetic field, the resonance condition becomes

$$
\omega-k_{\|} v_{\|}= \pm \frac{\Omega_{p}}{\gamma}
$$

where the upper and lower signs now refer to the L-mode and R-mode, respectively.

[14] For a given particle and ambient magnetic field, solutions for the resonant velocities $\left(v_{\perp}, v_{\|}\right)$require knowledge of the wave frequency, the wave number, and the wave polarization. In this study the wave frequency and polarization are measured quantities, whereas the wave number $k_{\|}$ must be determined by solving the appropriate cold plasma dispersion relation. The solution of the dispersion relation for a cold multi-ion plasma requires knowledge of the total ion composition which is first discussed below.

\subsection{Ion Composition}

[15] The total ion composition (thermal + higher energies) is dominated by the ion composition of the thermal plasma. However, routine ion measurements of the thermal plasma are problematic owing to experimental reasons. In particular, measurements outside of the plasmapause are affected by high positive spacecraft potentials and the nonequilibrium state of the lower density plasma [Young, 1983].

[16] Inside the plasmapause the relative concentrations of the thermal $(1-10 \mathrm{eV} / \mathrm{Q}) \mathrm{He}^{+}$ions and $\mathrm{O}^{+}$ions are typically of the order of 10-20\% [Young, 1983; Horwitz, 1987] and 1\% [Young et al., 1977; Young, 1983], respectively. At higher energies ( $\mathrm{E}>1 \mathrm{keV} / \mathrm{Q})$ the energy density of the ring current $\mathrm{He}^{+}$ions is found to be independent of magnetic activity and less than $2 \%$ of the total energy density [Daglis et al., 1999]. In contrast the energy density of the ring current $\mathrm{O}^{+}$ions is found to be highly variable ranging from $<5 \%$ of the total energy density during quiet times to $>60 \%$ during intense storms [Daglis et al., 1999].

[17] We require the total ion composition during storms, since EMIC waves are known to be most common at these times [Braysy et al., 1998; Erlandson and Ukhorskiy, 2001]. Unfortunately, there is very little information on the storm dependence of the total ion composition as a function of spatial location in the inner magnetosphere. Therefore we consider variations based on existing observations and implicitly assume that while major variations may occur in the energy density of the higher energy ions, the total ion composition is still dominated by the thermal ions. We choose an initial ion composition of $70 \% \mathrm{H}^{+}, 20 \% \mathrm{He}^{+}$, and 
$10 \% \mathrm{O}^{+}$(hereafter referred to as the storm-time ion composition) where the $\mathrm{He}^{+}$ion composition is based on the thermal ion composition [Young, 1983; Horwitz, 1987] and where we have increased the $\mathrm{O}^{+}$ion composition to $10 \%$ to take into account the potentially large increases in energetic $\mathrm{O}^{+}$which will contribute to an increase in the total $\mathrm{O}^{+}$ composition. We are not able to say by exactly how much the energetic $\mathrm{O}^{+}$increases the total $\mathrm{O}^{+}$composition. However, we test the sensitivity of the results to changes in the ion composition by repeating the analysis for a lowerpercentage heavy ion composition of $85 \% \mathrm{H}^{+}, 10 \% \mathrm{He}^{+}$, and $5 \% \mathrm{O}^{+}$(hereafter referred to as the nominal ion composition).

[18] Our results will show that the minimum resonant energies in a given band fall when the percentage ion composition in that band is increased. Thus larger percentage abundances of $\mathrm{H}^{+}\left(\mathrm{He}^{+}\right)$lead to lower minimum resonant energies for waves in the $\mathrm{H}^{+}\left(\mathrm{He}^{+}\right)$band. These results are consisitent with theoretical results [Summers and Thorne, 2003] which show that the electron minimum resonant energies depend significantly on the percentage density of the ion that specifies the band but are only weakly dependent on the other two percentage ion densities. Since most of the waves associated with the lower energy events will be found to lie in the "helium" band, the results will be most sensitive to the helium ion composition. There is only one event in the "oxygen" band, and this event is associated with minimum resonant energies greater than $20 \mathrm{MeV}$. Our results will therefore not be very sensitive to the $\mathrm{O}^{+}$ion composition. Indeed, moderate errors in the $\mathrm{O}^{+}$ion composition will result in relatively small errors in the $\mathrm{H}^{+}$and $\mathrm{He}^{+}$ion compositions, and these should be reasonably accounted for in our choice of variation for the ion compositions.

\subsection{Dispersion Relation}

[19] The dispersion relation for electromagnetic waves propagating in a uniform, cold plasma is given in many texts [e.g., Walker, 1993]. Specifically, the dispersion relation for parallel-propagating electromagnetic waves in a cold plasma containing a mixture of hydrogen, helium, and oxygen ions may be written as

$$
\frac{c^{2} k^{2}}{\omega^{2}}=1-\frac{\omega_{p e}^{2}}{\omega\left(\omega \mp\left|\Omega_{e}\right|\right)}-\sum_{l=1}^{3} \frac{\omega_{p l}^{2}}{\omega\left(\omega \pm \Omega_{l}\right)},
$$

where the upper sign combination refers to the R-mode, and the lower sign refers to the L-mode. The suffix $l$ denotes the ion species, with values $l=1,2$, and 3 referring to hydrogen, helium, and oxygen ions, respectively. Here, $\omega_{p l}=\sqrt{n_{l} q_{l}^{2} / \epsilon_{0} m_{l}}$ is the ion plasma frequency and $\Omega_{l}=q_{l} B_{0} / m_{l}$ is the ion gyrofrequency for each ion species, with $m_{1}=m_{p}, m_{2}=4 m_{p}$, and $m_{3}=16 m_{p}$.

[20] Figure 1a shows the dispersion relation for EMIC waves propagating parallel to the magnetic field. The electron gyrofrequency, $f_{c e}=\left|\Omega_{e}\right| / 2 \pi$, is set to $4 \mathrm{kHz}$ and the electron plasma frequency, $f_{p e}=\omega_{p e} / 2 \pi$, is set to $80 \mathrm{kHz}$, conditions that are typically encountered on the duskside around $L=6$. The dispersion characteristics of the L-mode waves are profoundly influenced by the presence of the $\mathrm{He}^{+}$ and $\mathrm{O}^{+}$ions, while the $\mathrm{R}$-mode waves are influenced only slightly. The presence of these additional heavy ions introduces two new resonances, at $\Omega_{\mathrm{He}^{+}}$, the helium ion gyrofrequency, and at $\Omega_{O+}$, the oxygen ion gyrofrequency. Two new cut-offs are introduced above the oxygen and helium ion gyrofrequencies, producing two stop-bands in which Lmode waves cannot propagate.

[21] Figure $1 \mathrm{~b}$ shows the dispersion relation for EMIC waves propagating at a moderate angle of $25^{\circ}$ to the ambient magnetic field. Here the heavy ions also lead to a polarization reversal, at two cross-over frequencies, where the waves become linearly polarized. With the exception of the regions close to each cross-over frequency, the phase velocity of EMIC waves is relatively insensitive to the angle of propagation for moderate propagation angles. Consequently, in the absence of specific information regarding the direction of propagation, the dispersion relation for fieldaligned waves (equation (4)) will be used in the following section to evaluate resonant particle energies.

\subsection{Calculation of the Minimum Resonant Energies}

[22] For prescribed wave and plasma conditions, solutions for the resonant particle velocities lie along semiellipses in the $\left(v_{\perp}, v_{\|}\right)$plane. For a fixed wave frequency the minimum resonant energy occurs when $v_{\perp}=0$. The resonance conditions in equation (2) or (3) can be solved using the expression for $k$ from the appropriate dispersion relation in equation (4) to yield an expression for the minimum parallel velocity

$$
v_{\|}=\frac{-y \pm \sqrt{y^{2}-4 x z}}{2 x}
$$

where $x=k^{2}+\Omega_{\sigma}^{2} / c^{2}, y=-2 \omega k, z=\omega^{2}-\Omega_{\sigma}^{2}$, and $\Omega_{\sigma}=\Omega_{e}$ for electrons and $\Omega_{\sigma}=\Omega_{p}$ for protons.

[23] For a given particle the two solutions are fed back into the appropriate resonance condition to determine the sign of the harmonic number. Consequently, for electrons the solution of the electron resonance condition (equation (2)), which yields $n=+1$, is appropriate for interaction with $\mathrm{R}$-mode waves, and the solution which yields $\mathrm{n}=-1$ is appropriate for interaction with L-mode waves. For protons the solution of the proton resonance condition (equation (3)), which yields $n=-1$ is appropriate for interaction with $\mathrm{R}$-mode waves, and the solution which yields $\mathrm{n}=+1$ is appropriate for interaction with L-mode waves. The minimum parallel resonant energy, $E_{\text {min }}$ is then determined from

$$
E_{\min }=(\gamma-1) m_{\sigma} c^{2}
$$

\section{CRRES Data Survey}

[24] CRRES measurements of the Poynting flux have shown that the magnetic equator is the favored region for EMIC wave generation [Fraser et al., 1996]. Therefore the observed wave frequencies are normalized to the equatorial proton gyrofrequency, which we determine from the local measurements by assuming a dipole magnetic field. These deduced equatorial gyrofrequencies are used to obtain a more realistic estimate of the minimum resonant energy. However, the particle plasma frequencies used in the energy calculations are the local values, since significant 

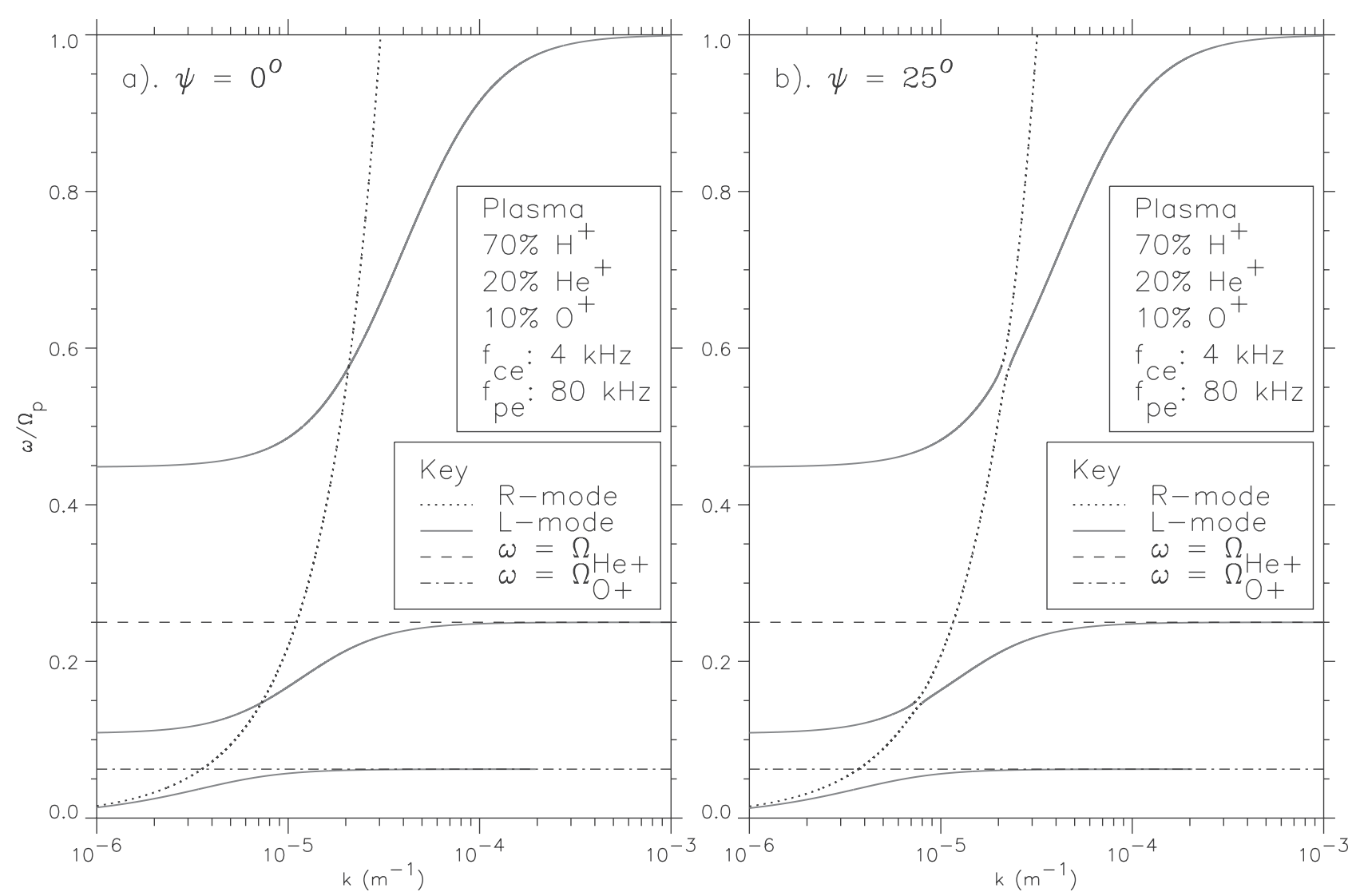

Figure 1. (a) The dispersion relation for electromagnetic waves propagating parallel to the ambient magnetic field in a plasma containing $70 \% \mathrm{H}^{+}, 20 \% \mathrm{He}^{+}$, and $10 \% \mathrm{O}^{+}$. The electron gyrofrequency is set to $4 \mathrm{kHz}$ and the electron plasma frequency is $80 \mathrm{kHz}$, conditions that typically occur on the dusk-side near $L=6$. (b) The dispersion relation for electromagnetic waves propagating at $25^{\circ}$ to the ambient magnetic field for the same conditions as in Figure 1a.

variation over the latitudes sampled by CRRES is unlikely. The L-mode and R-mode minimum resonant energies are calculated for both protons and electrons for each of the events in the database, at a time resolution of $8.2 \mathrm{~s}$, equal to the highest temporal resolution of the plasma frequency measurements.

[25] Figure 2 shows an example of a survey plot for orbit 968 as a function of UT between 1101 UT and 2007 UT on 30 August 1991. From bottom to top, the panels show the local number density, the ratio of the local electron plasma frequency to the local electron gyrofrequency, the local wave ellipticity, the local transverse power, the frequency normalised to the equatorial proton gyrofrequency, the Lmode (coded black) and R-mode (coded grey) proton minimum resonant energies, the L-mode (coded black) and R-mode (coded grey) electron minimum resonant energies, the $A E$ index and a bar plot of the $K p$ index, the Dst index, and, in the top panel, the solar wind velocity and IMF $\mathrm{Bz}$ determined from IMP 8 measurements.

[26] This event occurs during the main phase of a strong geomagnetic storm with a minimum Dst of $-107 \mathrm{nT}$ at 2100 UT. The CRRES apogee is near 1525 MLT, and there is no evidence during this orbit for a clear passage through the plasmapause. This is consistent with current models for the structure of the plasmasphere and the strong convective drift of thermal plasma towards the dayside magnetopause during a storm [Rasmussen et al., 1993]. EMIC wave events associated with electron minimum resonant energies less than $2 \mathrm{MeV}$ are located between $1330 \mathrm{UT}(L=6.0$, MLT $=$ $\left.1400, \lambda_{m}=-12.2^{\circ}\right)$ and $1820 \mathrm{UT}(L=5.3, \mathrm{MLT}=1706$, $\lambda_{m}=4.2^{\circ}$ ). In this region the waves are primarily lefthanded and cover a range of transverse powers from 1.0 to $20 \mathrm{nT}^{2} / \mathrm{Hz}$. During this orbit the electron minimum resonant energies fall below $2 \mathrm{MeV}$ for a total period of 1 hour and fall to below $1 \mathrm{MeV}$ for about 6 minutes. This example shows that the observed EMIC waves can resonate with $\sim \mathrm{MeV}$ electrons and hence can contribute to electron loss via pitch-angle scattering into the loss cone. Between 1330 UT and 1820 UT the proton minimum resonant energies are typically less than $10 \mathrm{keV}$, falling to values of the order of $2 \mathrm{keV}$ and $0.7 \mathrm{keV}$ during the periods when the minimum electron resonant energy falls to values of the order $2 \mathrm{MeV}$ and $1 \mathrm{MeV}$, respectively.

\section{Statistical Results}

[27] The case study presented above shows that the electron minimum resonant energy can fall into the important $\sim \mathrm{MeV}$ range. A comprehensive survey of all the EMIC wave events observed by CRRES has been undertaken to identify where and when these waves are most likely to contribute the loss of $\sim \mathrm{MeV}$ electrons. The spatial distri- 


\section{Summary Plot for CRRES Orbit 968}
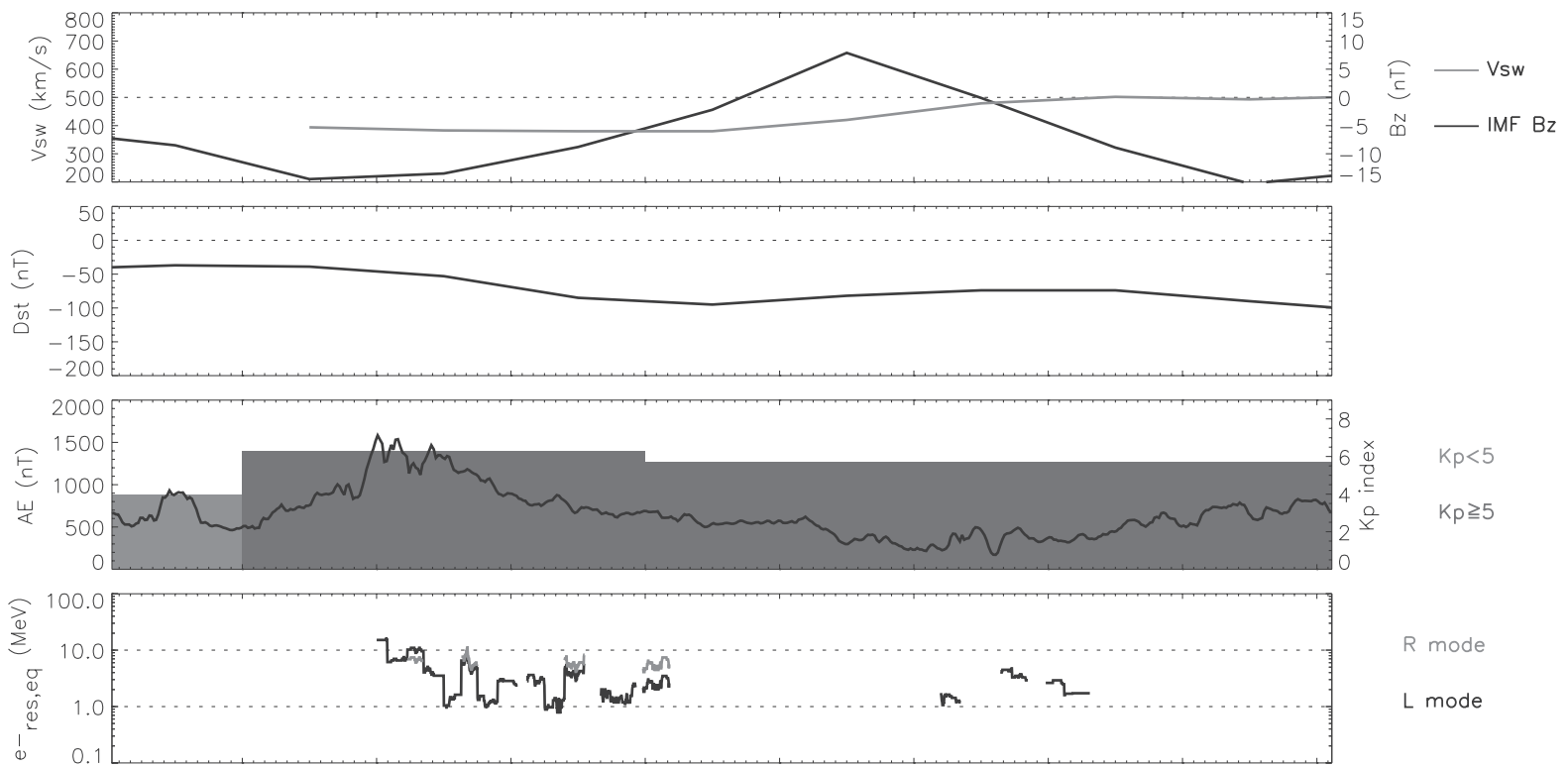

$R$ mode

$\mathrm{L}$ mode

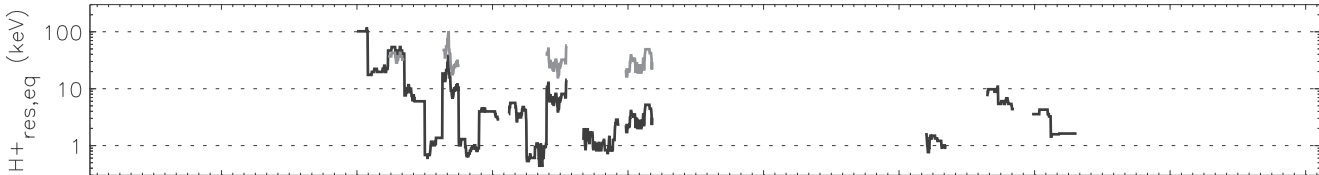

$\mathrm{R}$ mode

$\mathrm{L}$ mode

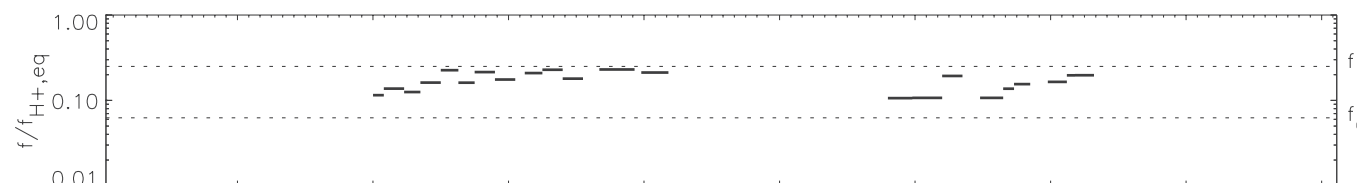

$\mathrm{fHe} /{ }^{\mathrm{f}} \mathrm{H}+, \mathrm{eq}$ $\mathrm{O}+/ \mathrm{f}_{\mathrm{H}} \mathrm{H}, \mathrm{eq}$
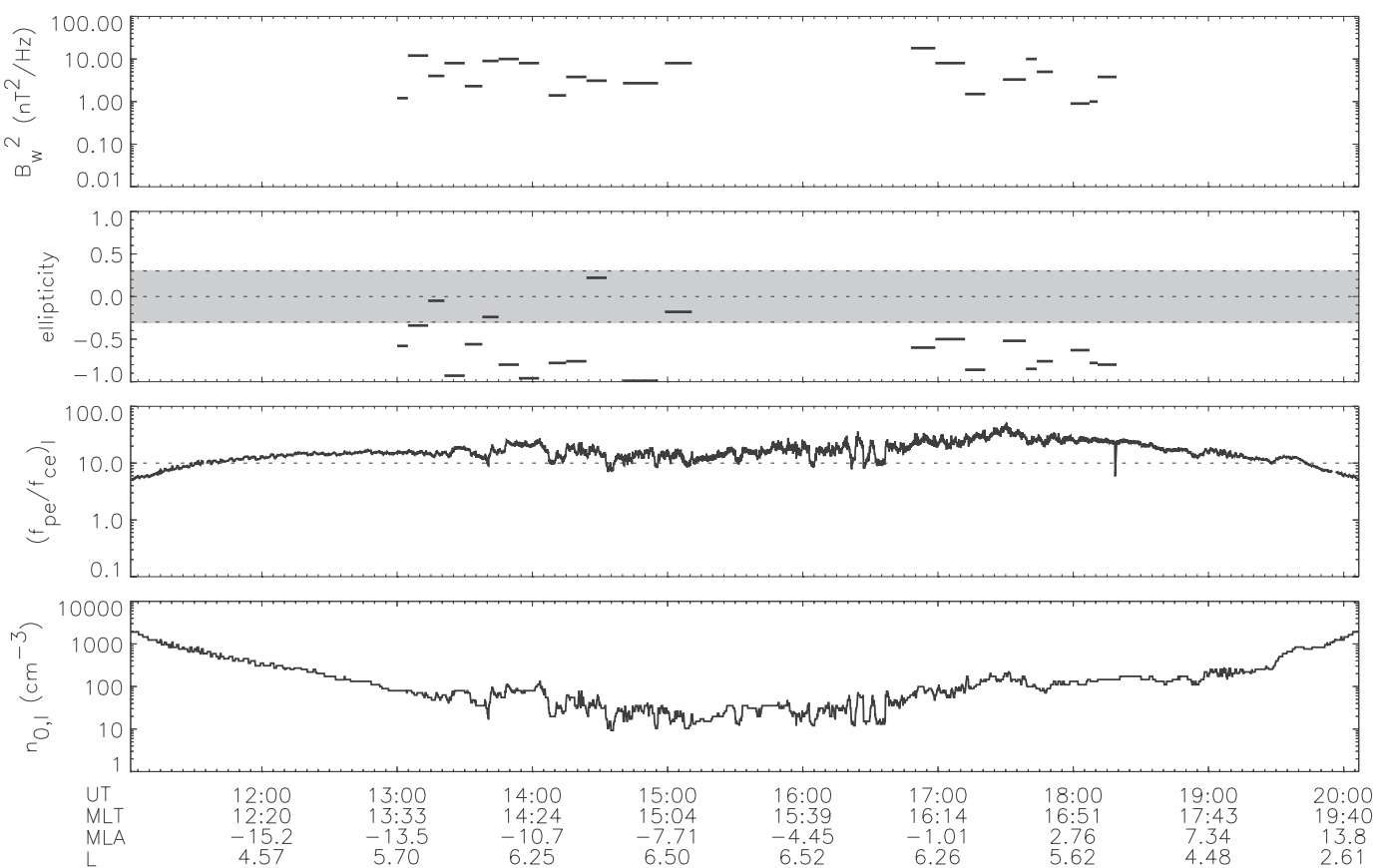

Figure 2. Summary plot of the relevant plasma and wave parameters for orbit 968 between 1101 and 2007 UT on 30 August 1991. The minimum resonant energies are calculated assuming the storm-time ion composition. 
bution of the observed events are first examined to identify the favored regions for EMIC waves. A detailed survey of the electron minimum resonant energies is then performed to identify the regions and the conditions under which the electron minimum resonant energies fall below $2 \mathrm{MeV}$. Finally, we examine the proton minimum resonant energies to identify the source particles of the EMIC waves likely to be associated with the precipitation of $\sim \mathrm{MeV}$ electrons.

\subsection{Spatial Distribution of the EMIC Wave Events}

[28] The top panel of Figure 3 shows the distribution of the EMIC wave events observed on CRRES as a function of $L$ and MLT. Here, noon is at the top, dawn is to the right, and the radial scale extends linearly out to $L=8$. The events are color-coded to show the ellipticity of the waves which are defined as being predominantly left-handed for $\epsilon<-0.3$ (coded black), approximately linear for $|\epsilon| \leq 0.3$ (coded green), and predominantly right-handed for $\epsilon>0.3$ (coded red). The majority of the EMIC wave events $(82 \%)$ are observed in the region between 1300 and 2000 MLT over the range of $L$ shells, $L>3$, as reported by Fraser and Nguyen [2001]. It should be noted that the occurrences are not normalized and that CRRES had limited coverage in the 0800-1400 local time sector due to the orbital coverage of CRRES during its operational lifetime. There are also difficulties in the identification of waves at lower $L(<3)$ due to the strong background magnetic field. The bottom panel of Figure 3 shows the distribution of the events in a meridian plane. Dipole field lines and lines of constant magnetic latitude are included on the plot to help visualize the location of the EMIC wave events as a function of $L, \lambda_{m}$. The events near the magnetic equator are mainly linear or left-hand polarized. There is a clear tendency for the polarization to become more linear with increasing magnetic latitude. The observation of a significant number of linear events $(|\epsilon| \leq 0.3)$ occurring near the equator is intriguing, since they cannot be explained by a polarization reversal from left-handed through linear to right-handed at the crossover frequency, as suggested by Young et al. [1981]. Rather, these linear polarized events may be due to a combination of both propagation and generation effects. The bias to negative magnetic latitudes is an orbital effect, caused by the fact that most of the events occurred in the afternoon sector when the spatial location of the CRRES spacecraft showed the same bias for $L>3$.

\subsection{Electron Minimum Resonant Energies}

[29] A survey of the L-mode and R-mode electron minimum resonant energies has been undertaken to identify the regions and conditions associated with electron minimum resonant energies below $2 \mathrm{MeV}$. In the absence of specific information on the direction of wave propagation, the dispersion relation in equation (4) for strictly parallel propagating EMIC waves is used to compute resonant energies. For consistency, only waves with a well defined polarization, namely $\mathrm{L}(\epsilon<-0.3)$ or $\mathrm{R}(\epsilon>0.3)$, are included in the survey. This yields a total of 416 events of well-defined polarization, the majority $(87 \%)$ of which are identified as L-mode. Two of these events occur at high latitude $\left(-25^{\circ}\right)$ with resulting frequencies normalized to the equatorial proton gyrofrequency lying within $0.5 \%$ of an ion gyrofrequency. These two events are excluded from the
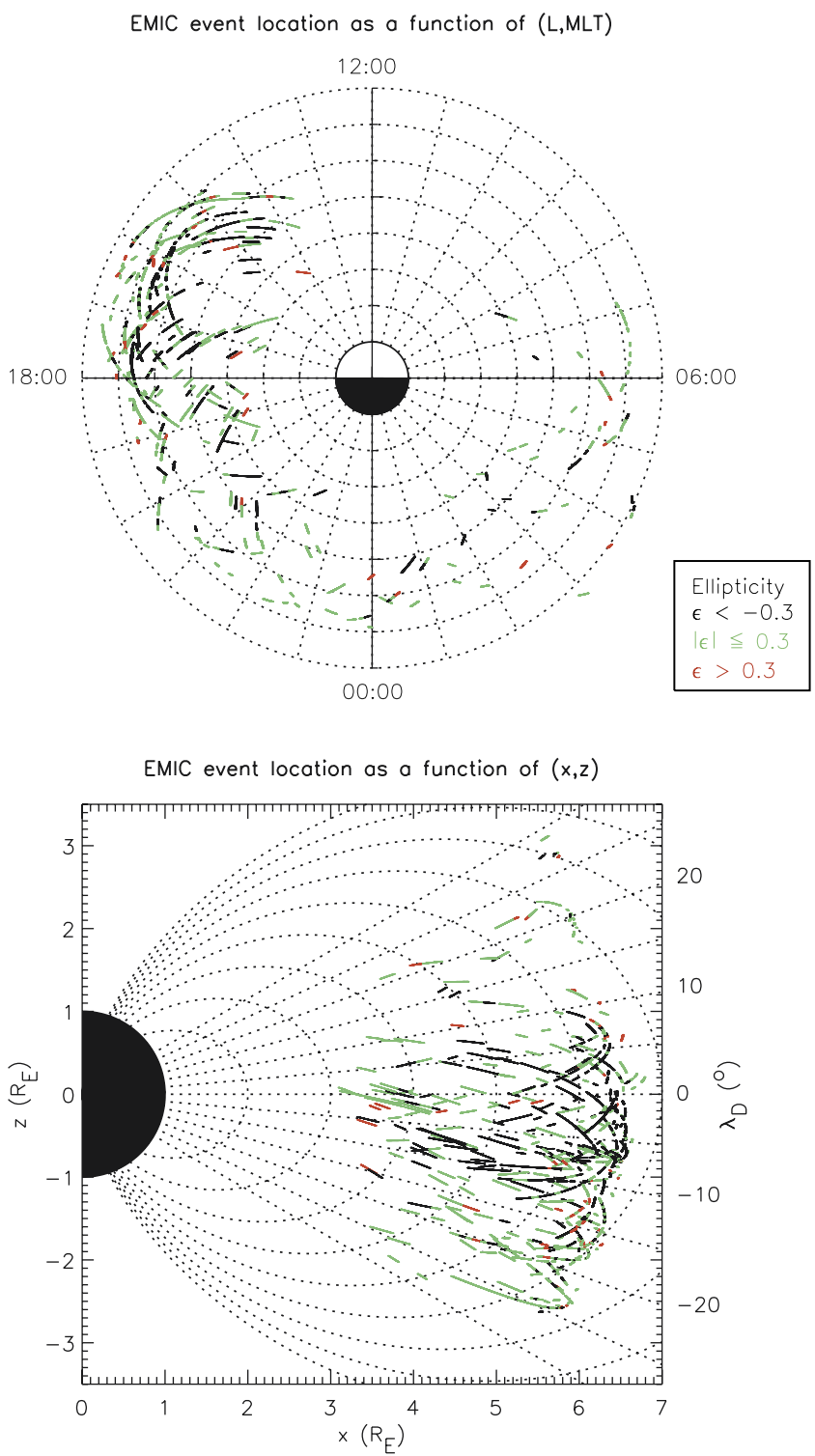

Figure 3. The distribution of the EMIC wave events as a function of $L$, MLT (top). It should be noted that the occurrences are not normalized and that CRRES had limited coverage in the 0800-1400 local time sector. The events are color-coded to show the ellipticity of the wave, which we define as being left-handed for $\epsilon<-0.3$ (coded black), linear for $|\epsilon| \leq 0.3$ (coded green), and right-handed for $\epsilon>0.3$ (coded red). The distribution of the events in a meridian plane (bottom). Dipole field lines and lines of constant magnetic latitude are included in the plot to help visualize the location of the EMIC wave events as a function of $\mathrm{L}, \lambda_{m}$.

survey since, in these particular circumstances, small errors in the derivation of the normalized frequency, caused for example by assuming a dipole field, can lead to very large errors in the minimum resonant energies.

\subsubsection{Spatial Distribution}

[30] The L-mode and R-mode electron minimum resonant energies for the storm-time ion composition $\left(70 \% \mathrm{H}^{+}, 20 \%\right.$ $\mathrm{He}^{+}$, and $10 \% \mathrm{O}^{+}$) are plotted as a function of $L$ shell in the top and bottom panels of Figure 4, respectively. The 

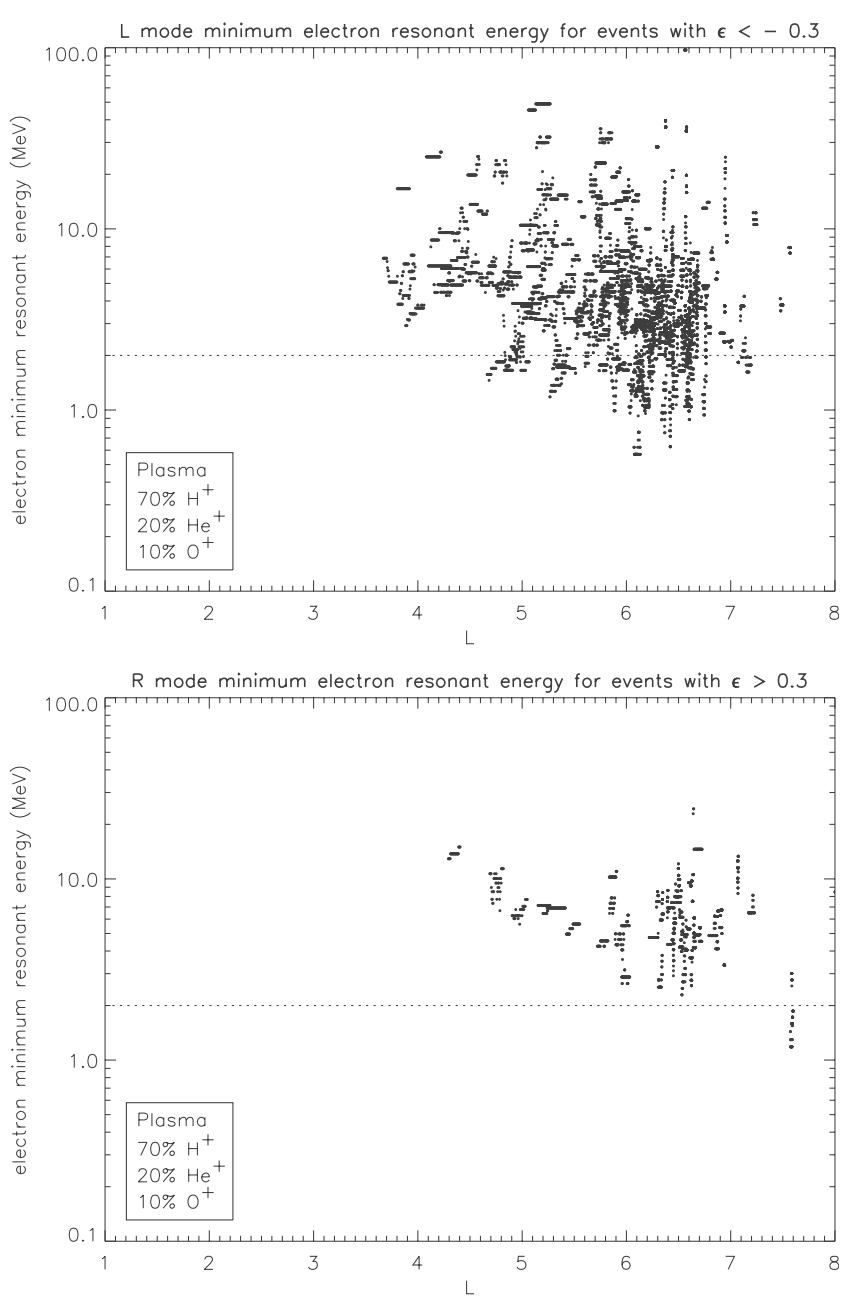

Figure 4. L-mode (top) and R-mode (bottom) electron minimum resonant energies for the storm-time ion composition as a function of $L$ shell.

L-mode electron minimum resonant energies exhibit considerable variability at any given $L$ shell. During a small fraction $(10.9 \%)$ of these L-mode observations, the minimum resonant energies fall below $2 \mathrm{MeV}$, these events all occurring in the region $L>4.5$. The R-mode electron minimum resonant energies tend to be greater than $2 \mathrm{MeV}$, but can sometimes fall to $\sim 2 \mathrm{MeV}$ at the highest $L$ shells sampled by CRRES $(L>6)$. Overall, during events of well-defined polarization, the resonant energies fall below $2 \mathrm{MeV}$ for $11.3 \%$ of the time. The majority $(92 \%)$ of these lower energy events are found to occur over a limited range of local times $(1300<$ MLT $<1800)$.

\subsubsection{Dependence on the Parameter $f_{p e} / f_{c e, e q}$}

[31] The dependence of the L-mode and R-mode electron minimum resonant energies on the ratio of the electron plasma frequency to the equatorial electron gyrofrequency $\left(f_{p e} / f_{c e, e q}\right)$ for the storm-time ion composition is shown in Figure 5. Each value of the ratio $f_{p e} / f_{c e, e q}$ is associated with a wide range of minimum resonant energies, but there is a clear systematic decrease in the minimum resonant energy as $f_{p e} / f_{c e, e q}$ increases. L-mode electron minimum resonant energies typically only fall to values below $2 \mathrm{MeV}$ for $f_{p e} / f_{c e, e q}>10$. Such conditions are found in regions of high plasma density and low magnetic field such as the duskside plasmapause or within detached plasma regions at high L. The R-mode electron minimum resonant energies fall below $2 \mathrm{MeV}$ only very occasionally but also when $f_{p e} / f_{c e, e q}>10$. The apparent gap in the R-mode electron minimum resonant energies is caused primarily by the small number of R-mode observations that occur in the range $0.2<\mathrm{f} / \mathrm{f}_{c p, e q}<0.3$.

5.2.3. Dependence on Frequency and Ion Composition [32] Much of the scatter exhibited in Figures 4 and 5 is associated with the strong dependence of the electron resonant energies on wave frequency. This dependence on frequency, $f$ (when normalized to the equatorial proton gyrofrequency, $\left.f_{c p, e q}\right)$ is shown explicitly in Figure 6 for the storm-time ion composition. The sensitivity of our results to the ion composition is examined in Figure 7 which shows the frequency dependence for the nominal ion composition. Decreasing the heavy ion composition tends to raise the L-mode electron minimum resonant energies in the "helium" band but reduces the L-mode electron minimum resonant energies in the "hydrogen" band.

[33] The L-mode electron minimum resonant energies are especially sensitive to the normalized wave frequency, and values below $2 \mathrm{MeV}$ occur in two distinct frequency ranges. In the "helium" band the minimum resonant energies can
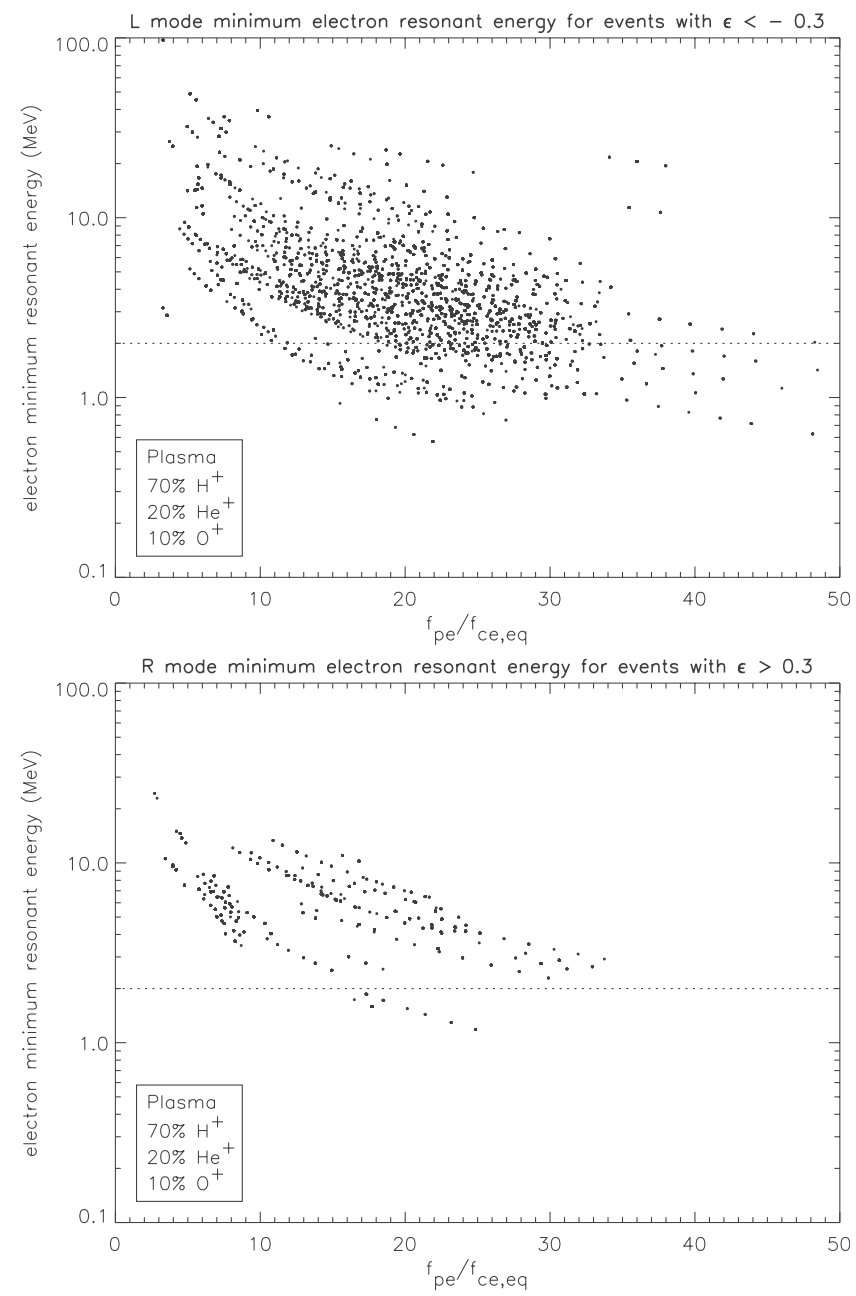

Figure 5. L-mode (top) and R-mode (bottom) electron minimum resonant energies for the storm-time ion composition as a function of $f_{p e} / f_{c e, e q}$. 

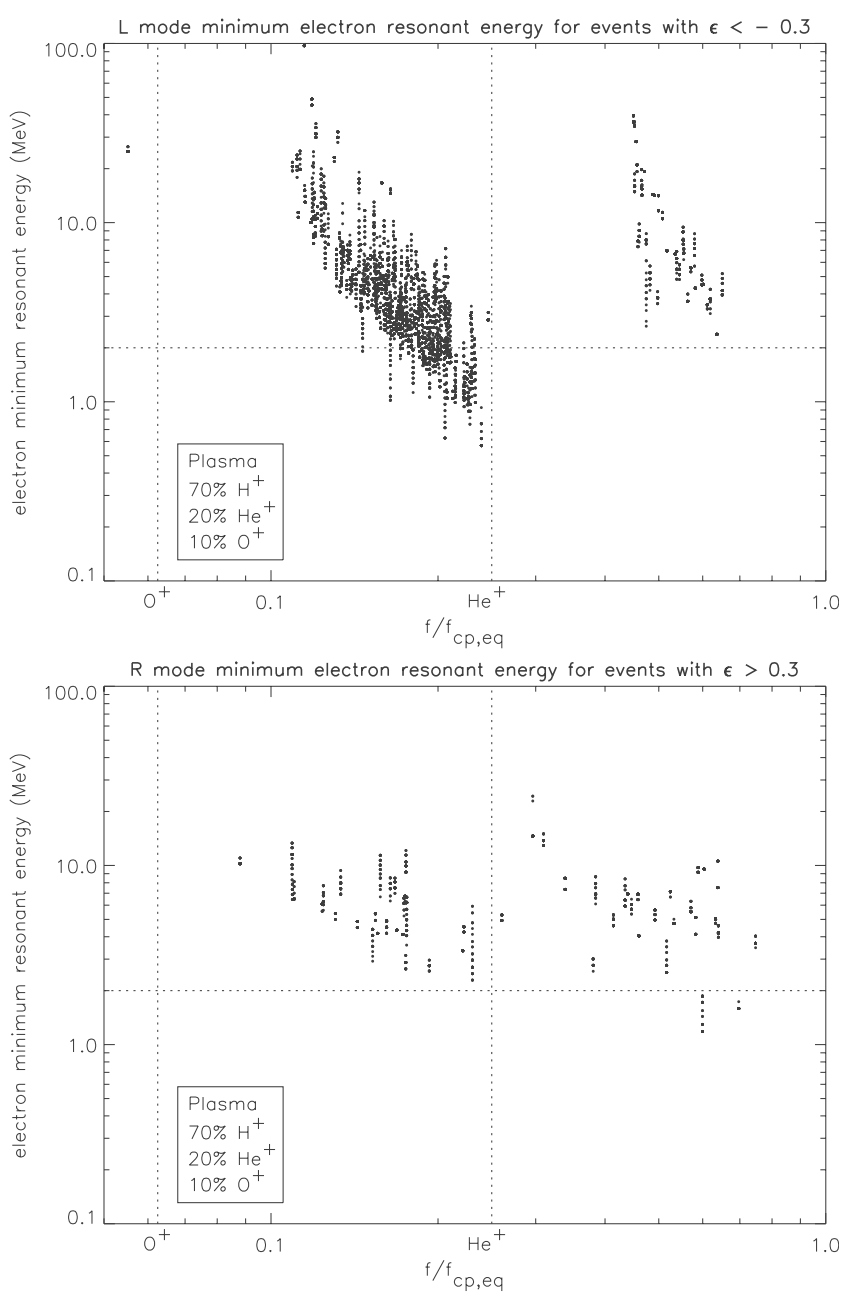

Figure 6. L-mode (top) and R-mode (bottom) electron minimum resonant energies as a function of wave frequency normalized to the equatorial proton gyrofrequency, assuming a storm-time ion composition with $70 \% \mathrm{H}^{+}, 20 \% \mathrm{He}^{+}$, and $10 \% \mathrm{O}^{+}$.

fall below $2 \mathrm{MeV}$ over a broad range of frequencies just below the helium ion gyrofrequency $\left(0.6 f_{\mathrm{cHe}^{+}}<f<f_{\mathrm{CHe}^{+}}\right)$. In the "hydrogen" band the minimum resonant energies fall below $2 \mathrm{MeV}$ over a range of frequencies just below the proton gyrofrequency $\left(0.4 f_{c p}<f<f_{c p}\right)$ but only for relatively low concentrations of the heavy ions (Figure 7). In contrast the R-mode electron minimum resonant energies rarely fall below $2 \mathrm{MeV}$, although minimum resonant energies of the order of $2 \mathrm{MeV}$ do occasionally occur for frequencies in the upper range of the "hydrogen" band $\left(0.6 f_{c p}<f<0.7 f_{c p}\right)$.

[34] In a given band the minimum resonant energies are found to be most sensitive to the percentage ion composition of the species that specifies the band and only weakly sensitive to the other two percentage ion compositions [Summers and Thorne, 2003]. A further test of the sensitivity of the derived minimum resonant energies to the ion composition in a given band can thus be obtained by plotting $E_{\text {min }}$ as a function of the percentage ion composition of the species that specifies the band. Therefore in Figure 8 we examine the sensitivity of the L-mode electron minimum resonant energies in each band $\left(\mathrm{H}^{+}, \mathrm{He}^{+}\right.$, and $\left.\mathrm{O}^{+}\right)$to the ion composition by plotting $E_{\min }$ as a function of the corresponding percentage ion composition $\left(\eta_{H^{+}}, \eta_{\mathrm{He}^{+}}\right.$, and $\left.\eta_{\mathrm{O}^{+}}\right)$for a set of frequencies just below the appropriate ion gyrofrequencies. We set $f_{p e} / f_{c e}$ to be 25 , which is a typical value associated with L-mode electron minimum resonant energies less than 2 $\mathrm{MeV}$. In the "hydrogen" band we set $\eta_{\mathrm{He}^{+}}=2 \eta_{\mathrm{O}_{+}}$, in the "helium" band we set $\eta_{O^{+}}=5 \%$, and in the "oxygen" band we set $\eta_{\mathrm{He}^{+}}=10 \%$, with $\eta_{\mathrm{H}^{+}}+\eta_{\mathrm{He}^{+}}+\eta_{\mathrm{O}^{+}}=100 \%$ at all times. In each band the minimum resonant energy for a fixed frequency is seen to fall with increasing percentage ion composition of the species that specifies the band.

[35] The L-mode electron minimum resonant energies in the "hydrogen" band are plotted as a function of the percentage $\mathrm{H}^{+}$composition in Figure 8a. The minimum resonant energies for waves with a normalized frequency of $f / f_{c p}=0.7$ lie below $2 \mathrm{MeV}$ for $\eta_{H^{+}}>42 \%$ whereas the minimum resonant energies for waves with a normalized frequency of $f / f_{c p}=0.5$ lie below $2 \mathrm{MeV}$ for $\eta_{H^{+}}>72 \%$. The L-mode electron minimum resonant energies in the "helium" band are plotted as a function of the percentage $\mathrm{He}^{+}$composition in Figure 8b. The minimum resonant
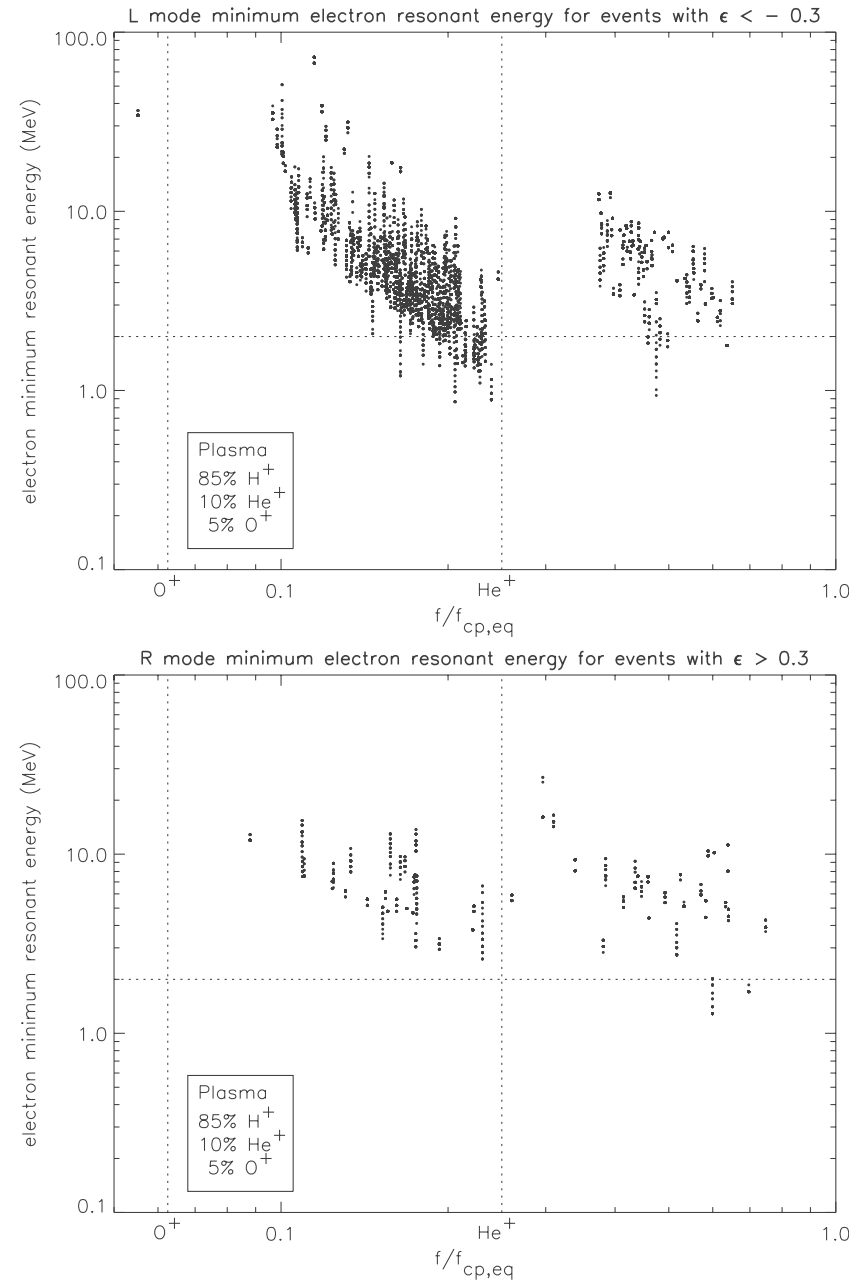

Figure 7. L-mode (top) and R-mode (bottom) electron minimum resonant energies as a function of wave frequency normalized to the equatorial proton gyrofrequency, assuming a lower heavy ion composition with $85 \% \mathrm{H}^{+}, 10 \% \mathrm{He}^{+}$, and $5 \% \mathrm{O}^{+}$. 

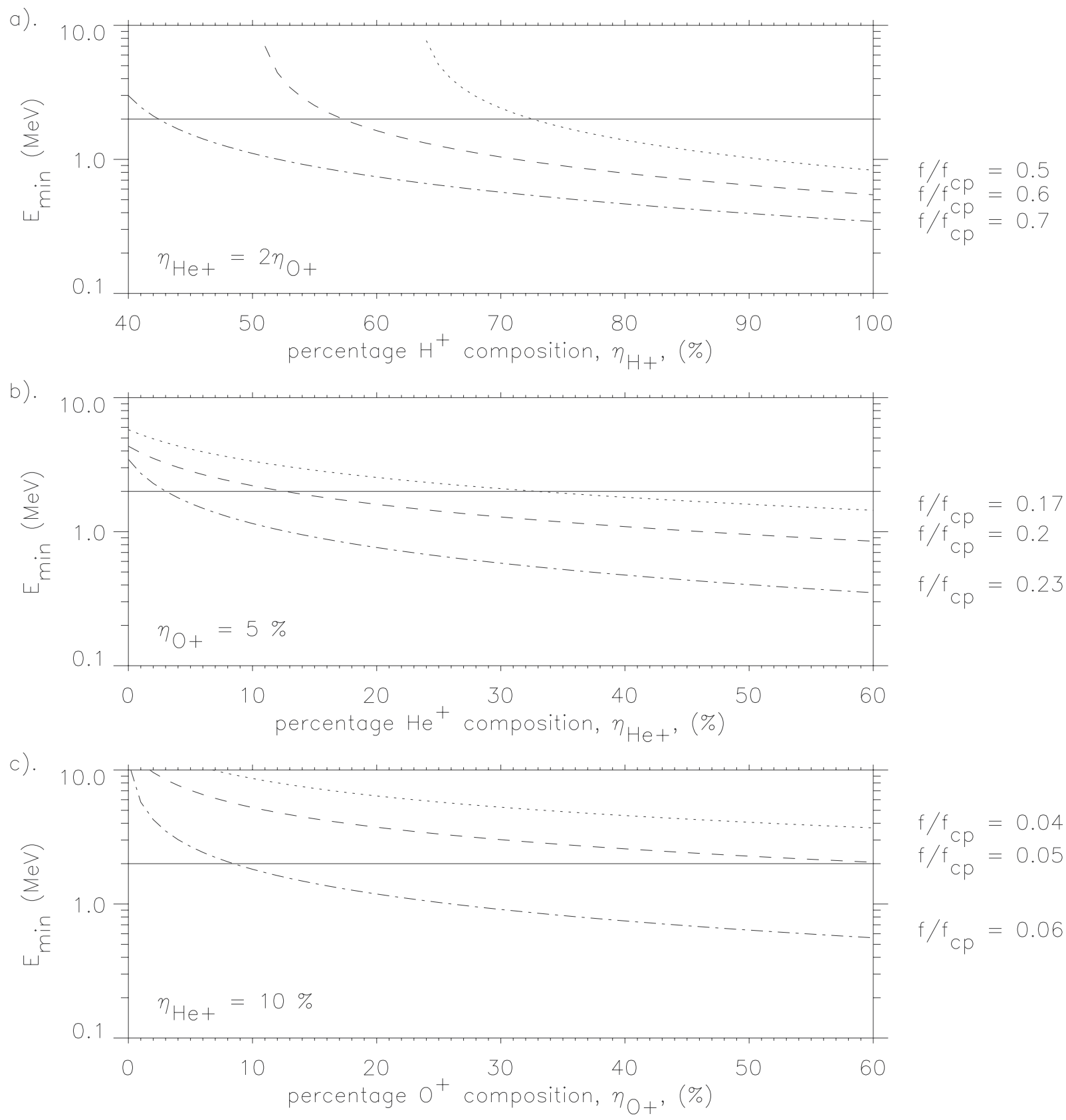

Figure 8. (a) L-mode electron minimum resonant energies for the specified wave frequencies in the "hydrogen" band as a function of the hydrogen ion composition. (b) L-mode electron minimum resonant energies for the specified wave frequencies in the "helium" band as a function of the helium ion composition. (c) L-mode electron minimum resonant energies for the specified wave frequencies in the "oxygen" band as a function of the oxygen ion composition. $\eta_{H^{+}}, \eta_{H_{e}+}$, and $\eta_{O+}$ are the percentage hydrogen, helium, and oxygen ion number densities, respectively, with $\eta_{\mathrm{H}^{+}}+\eta_{\mathrm{He}^{+}}+\eta_{\mathrm{O}^{+}}=100 \%$. The parameter $f_{p e} / f_{c e}$ is set at 25 , which is a typical value in regions where the L-mode electron minimum resonant energies fall below $2 \mathrm{MeV}$.

energies for waves with a normalized frequency of $f / f_{c p}=$ 0.23 lie below $2 \mathrm{MeV}$ for $\eta_{\mathrm{He}^{+}}>3 \%$ whereas the minimum resonant energies for waves with a normalized frequency of $f f f_{c p}=0.2$ lie below $2 \mathrm{MeV}$ for $\eta_{\mathrm{He}^{+}}>12 \%$. For the range of frequencies plotted there is a gradual reduction in $E_{\min }$ for $\eta_{\mathrm{He}^{+}}>10 \%$ with the minimum resonant energy typically falling by a factor of 2 between $\eta_{\mathrm{He}^{+}}=10 \%$ and $\eta_{\mathrm{He}^{+}}=$ $50 \%$. There is only one event in the "oxygen" band in our study but we have included a plot of the L-mode electron minimum resonant energies in the "oxygen" band as a function of the oxygen ion composition for completeness in Figure 8c. Here minimum resonant energies can fall below $2 \mathrm{MeV}$ at frequencies just below the oxygen ion gyrofrequency. For $\eta_{O_{+}}>10 \%$ there is a gradual reduction in $E_{\min }$ with the minimum resonant energy typically falling by a factor of 3 between $\eta_{O+}=10 \%$ and $\eta_{O+}=50 \%$. Most of the 

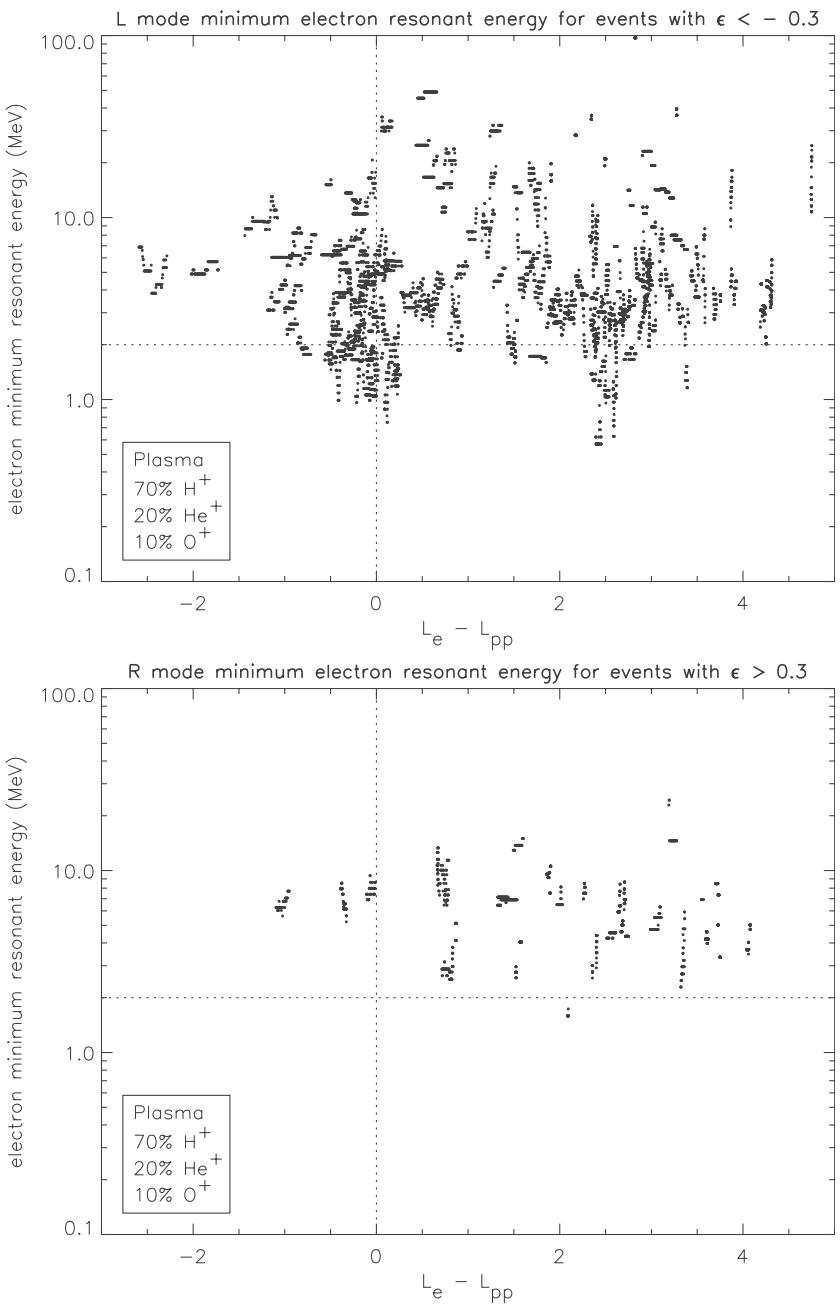

Figure 9. L-mode (top) and R-mode (bottom) electron minimum resonant energies for the storm-time ion composition as a function of distance from the observed plasmapause.

waves in our survey with minimum resonant energies less than $2 \mathrm{MeV}$ lie in the "helium" band and these results confirm that increasing the helium ion composition will result in a larger percentage of events being associated with minimum resonant energies less than $2 \mathrm{MeV}$.

\subsubsection{Dependence on Location With Respect to the Observed Plasmapause}

[36] The electron number density $\left(\mathrm{n}_{e}\right)$ profiles, in the same format as the bottom panel in Figure 2, were examined for each orbit, and a distinct plasmapause was identified if a change of $\mathrm{n}_{e}$ of greater than $10 \mathrm{~cm}^{-3}$ occurred over a radial distance of less than $0.5 \mathrm{R}_{e}$ [Fraser and Nguyen, 2001]. Orbits with multiple plasmapauses or density bite-outs were excluded from the analysis. The distance from the plasmapause $(\Delta L)$ was then determined for each event with a welldefined plasmapause by calculating the difference between the event location $\left(L_{e}\right)$ and the plasmapause position on the same inbound or outbound pass $\left(L_{p p}\right)$. Using this definition events with $\Delta L<0$ are inside the plasmapause and events with $\Delta L>0$ are outside the plasmapause. The electron minimum resonant energies are plotted as a function of $\Delta L$ for the storm-time ion composition in Figure 9. Regions associated with minimum resonant energies less than $2 \mathrm{MeV}$ occur predominantly in the vicinity of the plasmapause $(-0.75<\Delta L<0.25)$ and outside the plasmapause $(2<$ $\Delta L<3)$. It is interesting to note that these are also the regions associated with equatorial frequencies closest to the helium ion gyrofrequency [Fraser and Nguyen, 2001, Figure 13].

\subsubsection{Dependence on Magnetic Activity}

[37] The dependence of the electron minimum resonant energies on the geophysical indices $A E, K p$, and Dst have also been investigated. There is no clear dependence on either $A E$ or $K p$, although the L-mode electron minimum resonant energies rarely fall below $2 \mathrm{MeV}$ during magnetically quiet periods $(A E<100 \mathrm{nT}, K p<2)$. The minimum resonant energies, color-coded to show the storm phase, are plotted as a function of Dst index in Figure 10. Here all intervals with a Dst minimum of $-30 \mathrm{nT}$ or less were identified as storms and the recovery phase was defined to last until the Dst index returned to approximately $0 \mathrm{nT}$ or was interrupted by the arrival of a new storm. The L-mode electron minimum resonant energies may fall below $2 \mathrm{MeV}$ for almost any value of the Dst index, although there is a slight tendency for L-mode minimum resonant energies $<2 \mathrm{MeV}$ to occur during moderate or greater storms
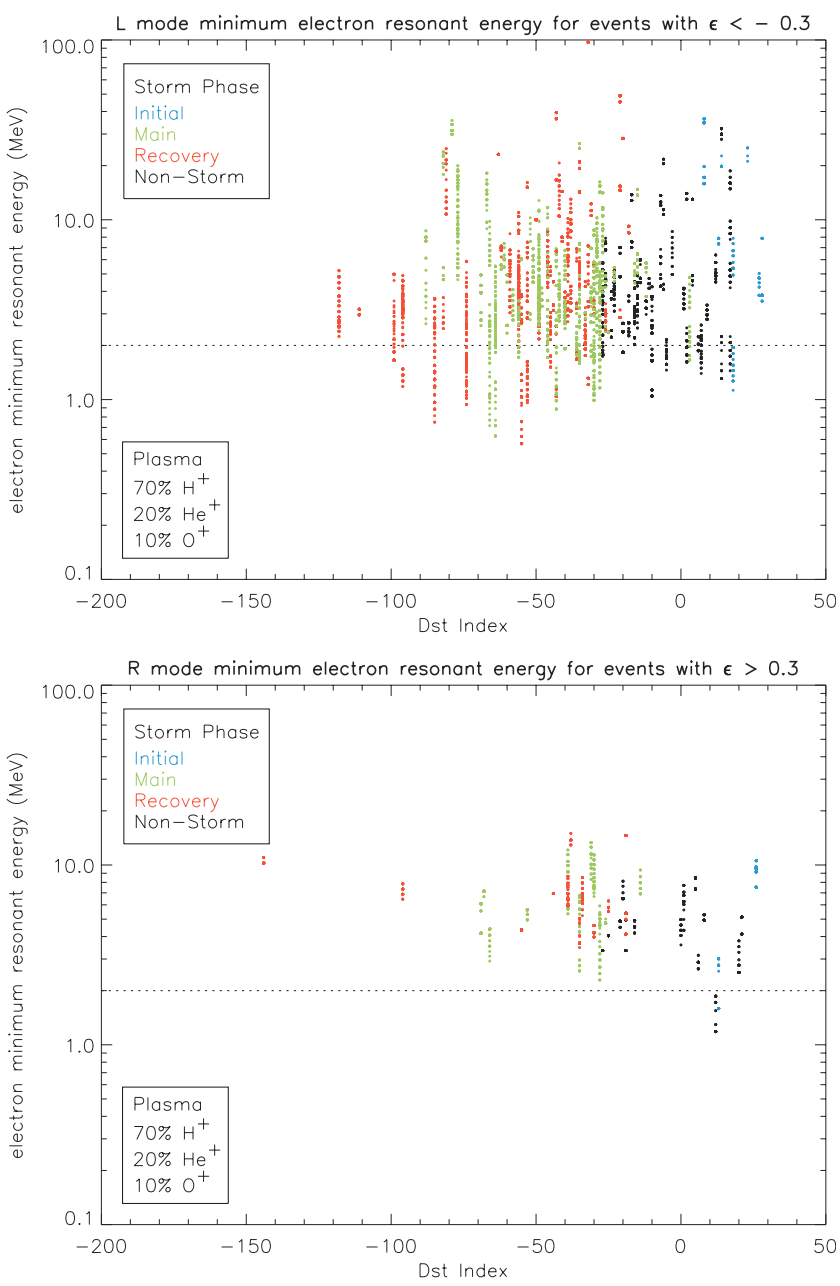

Figure 10. L-mode (top) and R-mode (bottom) electron minimum resonant energies for the storm-time ion composition as a function of Dst index. Each event is color-coded to show the storm-phase of the event. 
Table 1. Statistical Properties of the Events With $E_{\min }<2 \mathrm{MeV}$ for Storm-Time and Nominal Ion Compositions

\begin{tabular}{|c|c|c|c|c|c|}
\hline Conditions & Storm, $\mathrm{He}^{+}$ & Storm, $\mathrm{H}^{+}$ & Nominal, $\mathrm{He}^{+}$ & Nominal, $\mathrm{H}^{+}$ & Nominal, $\mathrm{H}^{+}$ \\
\hline Mode & L & $\mathrm{R}$ & $\mathrm{L}$ & L & $\mathrm{R}$ \\
\hline Number of events & 58 & 2 & 27 & 5 & 2 \\
\hline Number of readings & 2278 & 88 & 914 & 144 & 79 \\
\hline$\%$ time & 10.9 & 0.42 & 4.4 & 0.69 & 0.38 \\
\hline$\left\langle\mathrm{E}_{\min }\right\rangle, \mathrm{MeV}$ & 1.52 & 1.52 & 1.60 & 1.82 & 1.59 \\
\hline$\Delta \mathrm{E}_{\min }, \mathrm{MeV}$ & $0.57-2.0$ & $1.18-1.87$ & $0.87-2.0$ & $0.94-2.0$ & $1.29-1.86$ \\
\hline$\Delta\left(\mathrm{f} / \mathrm{f}_{c p, e q}\right)$ & $0.15-0.24$ & $0.60-0.70$ & $0.16-0.24$ & $0.46-0.64$ & $0.60-0.70$ \\
\hline$\langle\epsilon\rangle$ & -0.63 & 0.48 & -0.67 & -0.45 & 0.46 \\
\hline$\left\langle\mathrm{B}_{w}\right\rangle, \mathrm{nT}^{2} / \mathrm{Hz}$ & 4.15 & 0.25 & 5.2 & 0.85 & 0.27 \\
\hline$\Delta\left(\mathrm{f}_{p e} / \mathrm{f}_{c e, e q}\right)$ & $11.7-62.6$ & $16.5-24.9$ & $15.1-62.6$ & $10.9-33.2$ & $16.5-24.9$ \\
\hline$\Delta \mathrm{n}_{0}, \mathrm{~cm}^{-3}$ & $12.0-452.5$ & $13.18-35.24$ & $13.2-452.5$ & $9.31-89.0$ & $13.2-35.2$ \\
\hline$\Delta L$ & $4.66-7.2$ & $7.57-7.60$ & $4.68-6.77$ & $6.11-7.57$ & $7.57-7.60$ \\
\hline$\langle|\lambda|\rangle$, deg & 9.5 & 23.7 & 8.3 & 20.3 & 23.72 \\
\hline$\Delta$ MLT, am & $0016-0445$ & - & $0100-0444$ & $0432-0434$ & - \\
\hline$\Delta \mathrm{MLT}, \mathrm{pm}$ & $1330-2120$ & $1615-1633$ & $1330-2108$ & $1352-1559$ & $1615-1633$ \\
\hline$\langle A E\rangle, \mathrm{nT}$ & 568 & 226 & 658 & 293 & 236 \\
\hline$\langle K p\rangle$ & 4.7 & 3.7 & 5.0 & 3.7 & 3.7 \\
\hline$\Delta D s t, \mathrm{nT}$ & $-99-18$ & $12-13$ & $-96-18$ & $-88-28$ & $12-13$ \\
\hline
\end{tabular}

$(D s t<-50 \mathrm{nT})$. The majority (84\%) of the lower-energy events occur during geomagnetic storms, although lowerenergy events may also occur outside of storm periods. Indeed, the L-mode minimum electron resonant energies may also fall below $2 \mathrm{MeV}$ during the initial phase of a geomagnetic storm, when the Dst index is positive.

\subsubsection{Subset of EMIC Events With $E_{\text {min }}<2 \mathrm{MeV}$}

[38] The statistical properties of geophysically interesting events with electron minimum resonant energies below 2 $\mathrm{MeV}$ are listed in Table 1. This small but important subset of all CRRES EMIC events is associated with relatively high plasma density $\left(9-452 \mathrm{~cm}^{-3}\right)$ typical of the afternoon bulge region of the outer plasmasphere. The events are predominantly L-mode waves that propagate in the band just below the helium gyrofrequency and tend to occur during periods of high geomagnetic activity. The average wave power spectral intensity $\left(4-5 \mathrm{nT}^{2} / \mathrm{Hz}\right)$, coupled with typical bandwidths of the order of $0.3 \mathrm{~Hz}$, lead to wide band amplitudes of the order of $1 \mathrm{nT}$, which are comparable to the requirement for strong diffusion scattering [Thorne and Kennel, 1971; Summers and Thorne, 2003].

\subsection{Proton Minimum Resonant Energies}

[39] The CRRES data base can also be used to evaluate proton minimum resonant energies with both L-mode and R-mode waves. In particular we are interested in the proton minimum resonant energies associated with electron minimum resonant energies below $2 \mathrm{MeV}$. A comparison between the proton and electron minimum resonant energies for the storm-time ion composition is given in Figure 11 for L-mode waves (top panel) and R-mode waves (bottom panel). L-mode electron minimum resonant energies below $2 \mathrm{MeV}$ are associated with proton minimum resonant energies below $2 \mathrm{keV}$. The R-mode electron minimum resonant energies are generally above $2 \mathrm{MeV}$ but may fall to the order of $2 \mathrm{MeV}$ for $\mathrm{R}$-mode proton minimum resonant energies in the range of $5-10 \mathrm{keV}$. These results suggest that the EMIC waves which resonate with the $\sim \mathrm{MeV}$ electrons are produced by low-energy $(\sim \mathrm{keV})$ protons.

\section{Conclusions}

[40] We have conducted a comprehensive statistical survey of the electron and proton minimum resonant energies for cyclotron resonance with L-mode and R-mode EMIC waves observed on the CRRES spacecraft. Over 800 EMIC wave events were initially identified during the CRRES mission, the majority of which occurred on the duskside of the magnetosphere $(1300<$ MLT $<2000)$ for $L>3$. Minimum resonant energies were then calculated for a
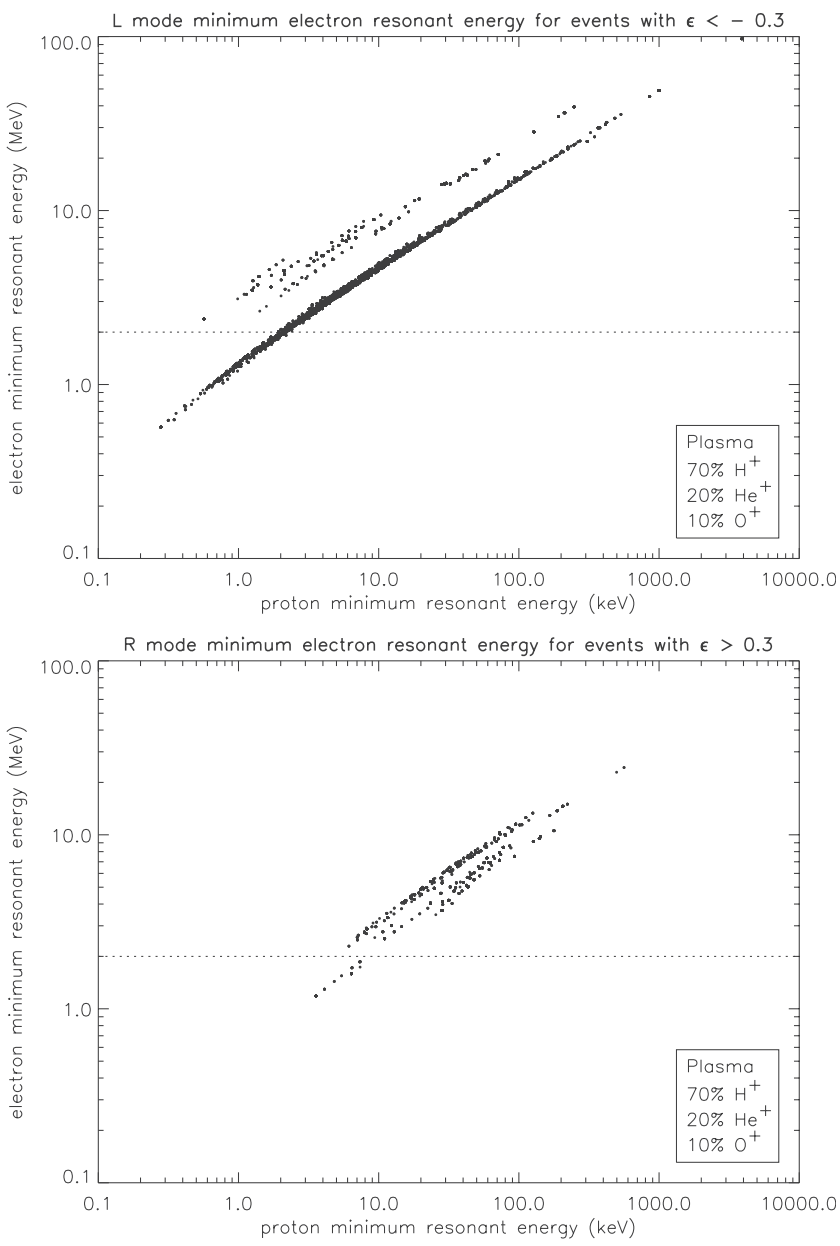

Figure 11. A comparison between electron and proton minimum resonant energies for the storm-time ion composition for L-mode waves (top), and R-mode waves (bottom). 
subset of over 400 EMIC events with well defined polarization. The principal results of the analysis are as follows:

[41] 1. Electron minimum resonant energies with observed EMIC waves span a broad range $(500 \mathrm{keV}<\mathrm{E}<$ $50 \mathrm{MeV}$ ) and are found to fall into the geophysically important range below $2 \mathrm{MeV}$ during a small fraction $(\sim 11.3 \%)$ of the observations. These lower energy events are primarily associated with resonant L-mode waves and occur over a broad range of $L$ shells $(L>4.5)$ and local times $(1300<$ MLT $<1800)$.

[42] 2. Electron minimum resonant energies of less than $2 \mathrm{MeV}$ are restricted to regions of high plasma density and/or low magnetic field, where $f_{p e} / f_{c e, e q}>10$.

[43] 3. Electron minimum resonant energies depend strongly on wave frequency. In particular, resonance below $2 \mathrm{MeV}$ is primarily associated with EMIC waves just below the helium and proton gyrofrequencies.

[44] 4. For the limited subset of L-mode events with minimum resonant electron energies below $2 \mathrm{MeV}$, the average wave spectral intensity is $4-5 \mathrm{nT}^{2} / \mathrm{Hz}$, which is sufficient to cause electron pitch-angle diffusion near the strong diffusion limit.

[45] 5. The EMIC waves which resonate with the $\sim \mathrm{MeV}$ electrons are produced by low-energy $(\sim \mathrm{keV})$ protons.

[46] If relativistic electrons were continuously subject to strong diffusion wave scattering, the effective lifetime for precipitation loss to the atmosphere would be several minutes [e.g., Thorne and Kennel, 1971]. This is much faster than any hypothesized acceleration mechanism, and such catastrophic loss would lead to a rapid depletion of the outer radiation zone. Our analysis of the CRRES data has shown that there is a limited subset of observed EMIC waves that are able to resonate with $\leq 2 \mathrm{MeV}$ electrons and that these waves are primarily confined to a restricted range of local times. The average power spectral intensity of this geophysically important subset is sufficient to cause strong diffusion scattering. This suggests that $\leq 2 \mathrm{MeV}$ relativistic electrons are only subject to strong diffusion scattering over a small fraction of their drift orbit. Driftaveraged scattering lifetimes will therefore lie in the range of several hours to a day, as initially pointed out in the theoretical study by Summers and Thorne [2003]. Such a time-scale is still short compared to the duration of a storm, and it suggests that EMIC waves should play an important role in relativistic electron loss during storm conditions. The EMIC waves themselves are most likely to be generated by anisotropic distributions of low-energy $(\sim \mathrm{keV})$ protons which should be also precipitated along with the $\sim \mathrm{MeV}$ electrons.

[47] Acknowledgments. We thank the World Data Centre C1 for STP at the Rutherford Appleton Laboratory and the NSSDC Omniweb for providing the geomagnetic indices and solar wind parameters used in this paper. This research was funded in part by NASA grant NAG5-11922. D. S. acknowledges support from the Natural Sciences and Engineering Research Council of Canada under grant A-0621.

Lou-Chuang Lee thanks Kirsten R. Lorentzen and Reiner H. W. Friedel for their assistance in evaluating this paper.

\section{References}

Anderson, B. J., R. E. Erlandson, and L. J. Zanetti, A statistical study of Pc 1-2 magnetic pulsations in the equatorial magnetosphere: 1. Equatorial occurrence distributions, J. Geophys. Res., 97, 3075, 1992a.
Anderson, R. R., D. A. Gurnett, and D. L. Odem, CRRES plasma wave experiment, J. Spacecr. Rockets, 29, 570, 1992 b.

Baker, D. N., S. Kanekal, J. B. Blake, B. Klecker, and G. Rostoker, Satellite anomalies linked to electron increase in the magnetosphere, Eos Trans. $A G U, 75,401,1994$.

Baker, D. N., J. H. Allen, S. G. Kanekal, and G. D. Reeves, Disturbed space environment may have been related to satellite pager failure, Eos Trans. $A G U, 79,477,1998$.

Bossen, M., R. L. McPherron, and C. T. Russell, A statistical study of Pc 1 magnetic pulsations at synchronous orbit, J. Geophys. Res., 81, 6083, 1976.

Braysy, T., K. Mursula, and G. Markland, Ion cyclotron waves during a great geomagnetic storm observed by Freja double-probe electric field instrument, J. Geophys. Res., 103, 4145, 1998.

Cornwall, J. M., F. V. Coroniti, and R. M. Thorne, Turbulent loss of ring current protons, J. Geophys. Res., 75, 4699, 1970.

Daglis, I. A., R. M. Thorne, W. Baumjohann, and S. Orsini, The terrestrial ring current: Origin formation and decay, Rev. Geophys., 37, 407, 1999.

Desorgher, L., P. Bühler, A. Zehnder, and E. O. Flückiger, Simulations of the outer radiation belt flux decrease during the March 26, 1995, magnetic storm, J. Geophys. Res., 105, 21,211, 2000.

Elkington, S. R., M. K. Hudson, and A. A. Chan, Acceleration of relativistic electrons via drift-resonant interaction with toroidal-mode Pc-5 ULF oscillations, Geophys. Res. Lett., 26, 3273, 1999.

Erlandson, R. E., and A. J. Ukhorskiy, Observations of electromagnetic ion cyclotron waves during geomagnetic storms: Wave occurrence and pitch angle scattering, J. Geophys. Res., 106, 3883, 2001.

Foat, J. E., R. P. Lin, D. M. Smith, F. Fenrich, R. Milan, I. Roth, K. R. Lorentzen, M. P. McCarthy, G. K. Parks, and J. P. Treilhou, First detection of a terrestrial MeV X-ray burst, Geophys. Res. Lett., 25, 4109, 1998.

Fraser, B. J., and R. L. McPherron, Pc 1-2 magnetic pulsation spectra and heavy ion effects at synchronous orbit: ATS 6 results, J. Geophys. Res., $87,4560,1982$.

Fraser, B. J., and T. S. Nguyen, Is the plasmapause a preferred source region of electromagnetic ion cyclotron waves in the magnetosphere?, J. Atmos. Terr. Phys., 63, 1225, 2001.

Fraser, B. J., H. J. Singer, W. J. Hughes, J. R. Wygant, R. R. Anderson, and D. Y. Hu, CRRES Poynting vector observations of electromagnetic ion cyclotron waves near the plasmapause, J. Geophys. Res., 101, 15,331, 1996.

Friedel, R. H. W., G. D. Reeves, and T. Obara, Relativistic electron dynamics in the inner magnetosphere-A review, J. Atmos. Sol. Terr. Phys., $64,265,2002$

Gurnett, D. A., and R. R. Shaw, Electromagnetic radiation trapped in the magnetosphere above the plasma frequency, J. Geophys. Res., 78, 8136, 1973.

Horne, R. B., and R. M. Thorne, On the preferred source location for the convective amplification of ion cyclotron waves, J. Geophys. Res., 98, 9233, 1993

Horne, R. B., and R. M. Thorne, Convective instabilities of electromagnetic ion cyclotron waves in the outer magnetosphere, J. Geophys. Res., 99, $17,259,1994$.

Horne, R. B., and R. M. Thorne, Potential waves for relativistic electron scattering and stochastic acceleration during magnetic storms, Geophys. Res. Lett., 25, 3011, 1998.

Horne, R. B., N. P. Meredith, R. M. Thorne, D. Heynderickx, R. H. A. Iles, and R. R. Anderson, Evolution of energetic pitch angle distributions during storm-time electron acceleration to megaelectronvolt energies, J. Geophys. Res., 108(A1), 1016, doi:10.1029/2001JA009165, 2003.

Horwitz, J. L., Core plasma in the magnetosphere, Rev. Geophys., 25, 3579, 1987.

Hudson, M. K., S. R. Elkington, J. G. Lyon, M. J. Wiltberger, and M. Lessard, Radiation belt electron acceleration by ULF wave drift resonance: Simulation of 1997 and 1998 storms, in Space Weather, Geophys. Monogr. Ser., vol. 125, edited by P. Song, H. J. Singer, and G. L. Siscoe, p. 289, AGU, Washington, D.C., 2001.

Iles, R. H. A., A. N. Fazakerley, A. D. Johnstone, N. P. Meredith, and P. Bühler, The relativistic electron response in the outer radiation belt during magnetic storms, Ann. Geophys., 20, 957, 2002.

Jordanova, V. K., C. J. Farrugia, R. M. Thorne, G. V. Khazanov, G. D. Reeves, and M. F. Thomsen, Modeling ring current proton precipitation by electromagnetic ion cyclotron waves during the May 14-16, 1997, storm, J. Geophys. Res., 106, 7, 2001.

Kim, H.-J., and A. A. Chan, Fully adiabatic changes in storm time relativistic electron fluxes, J. Geophys. Res., 102, 22,107, 1997.

Kozyra, J. U., T. E. Cravens, A. F. Nagy, E. G. Fontheim, and R. S. B. Ong, Effects of energetic heavy ions on electromagnetic ion cyclotron wave generation in the plasmapause region, J. Geophys. Res., 89, 2217, 1984. $\mathrm{Li}, \mathrm{X}$., and M. A. Temerin, The electron radiation belt, Space Sci. Rev., 95, 569,2001 
Li, X., D. N. Baker, M. Temerin, T. E. Cayton, G. D. Reeves, R. A Christiansen, J. B. Blake, M. D. Looper, R. Nakamura, and S. G. Kanekal, Multi-satellite observations of the outer zone electron variation during the November 3-4, 1993, magnetic storm, J. Geophys. Res., 102, 14,123, 1997.

Lorentzen, K. R., M. P. McCarthy, G. K. Parks, J. E. Foat, R. M. Milan, D. M. Smith, R. P. Lin, and J. P. Treilhou, Precipitation of relativistic electrons by interaction with electromagnetic ion cyclotron waves, J. Geophys. Res., 105, 5381, 2000.

Lorentzen, K. R., J. B. Blake, U. S. Inan, and J. Bortnik, Observations of relativistic electron microbursts in association with VLF chorus, J. Geophys. Res., 106, 6017, 2001.

Lyons, L. R., and R. M. Thorne, Parasitic pitch angle diffusion of radiation belt particles by ion cyclotron waves, J. Geophys. Res., 77, 5608, 1972.

Mathie, R. A., and I. R. Mann, On the solar wind control of Pc5 ULF pulsation power at mid-latitudes: Implications for $\mathrm{MeV}$ electron acceleration in the outer radiation belt, J. Geophys. Res., 106, 29,783, 2001.

Meredith, N. P., R. B. Horne, and R. R. Anderson, Substorm dependence of chorus amplitudes: Implications for the acceleration of electrons to relativistic energies, J. Geophys. Res., 106, 13,165, 2001.

Meredith, N. P., R. B. Horne, R. H. A. Iles, R. M. Thorne, D. Heynderickx, and R. R. Anderson, Outer zone relativistic electron acceleration associated with substorm-enhanced whistler mode chorus, J. Geophys. Res., 107(A7), 1144, 10.1029/2001JA900146, 2002a.

Meredith, N. P., R. B. Horne, D. Summers, R. M. Thorne, R. H. A. Iles, D. Heynderickx, and R. R. Anderson, Evidence for acceleration of outer zone electrons to relativistic energies by whistler mode chorus, Ann. Geophys., 20, 967, 2002b.

Meredith, N. P., M. Cain, R. B. Horne, R. M. Thorne, D. Summers, and R. R. Anderson, Evidence for chorus-driven electron acceleration to relativistic energies from a survey of geomagnetically-disturbed periods, J. Geophys. Res., 108, doi:10.1029/2002JA009764, in press, 2003.

Millan, R. H., R. P. Lin, D. M. Smith, K. R. Lorentzen, and M. P. McCarthy, X-ray observations of $\mathrm{MeV}$ electron precipitation with a balloon-borne germanium spectrometer, Geophys. Res. Lett., 29(24), 2194, doi:10.1029/2002GL015922, 2002.

Miyoshi, Y., A. Morioka, T. Obara, H. Misawa, T. Nagai, and Y. Kasahara, Rebuilding process of the outer radiation belt during the November 3, 1993 magnetic storm-NOAA and EXOS-D observations, J. Geophys. Res., 108(A1), 1004, doi:10.1029/2001JA007542, 1993.

O'Brien, T. P., R. L. McPherron, D. Sornette, G. D. Reeves, R. Friedel, and H. Singer, Which magnetic storms produce relativistic electrons at geosynchronous orbit?, J. Geophys. Res., 106, 15,533, 2001.

Perraut, S., R. Gendrin, and A. Roux, Amplification of ion cyclotron waves for various typical radial profiles of magnetospheric parameters, J. Atmos. Terr. Phys., 38, 1191, 1976.

Rasmussen, C. E., S. M. Guiter, and S. G. Thomas, Two-dimensional model of the plasmasphere, Planet. Space Sci., 41, 35, 1993.

Reeves, G. D., K. L. McAdams, R. H. W. Friedel, and T. P. O'Brien, Acceleration and loss of relativistic electrons during geomagnetic storms, Geophys. Res. Lett., 29, 35, doi:10.1029/2002GL016513, in press, 2003.

Roux, A., S. Perraut, J. L. Rauch, C. de Villedary, G. Kremser, A. Korth, and D. T. Young, Wave-particle interactions near $\Omega_{\mathrm{H}^{+}}$observed on board GEOS 1 and 2: 2. Generation of ion cyclotron waves and heating of $\mathrm{He}^{+}$ ions, J. Geophys. Res., 87, 8174, 1982.

Singer, H. J., W. P. Sullivan, P. Anderson, F. Mozer, P. Harvey, J. Wygant, and W. McNeil, Fluxgate magnetometer instrument on the CRRES, J. Spacecr. Rockets, 29, 599, 1992.

Summers, D., and C.-Y. Ma, A model for generating relativistic electrons in the Earth's inner magnetosphere based on gyroresonant wave-particle interactions, J. Geophys. Res., 105, 2625, 2000 a.

Summers, D., and C.-Y. Ma, Rapid acceleration of electrons in the magnetosphere by fast-mode MHD waves, J. Geophys. Res., 105, 15,887, 2000 b.
Summers, D., and R. M. Thorne, Relativistic electron pitch-angle scattering by electromagnetic ion cyclotron waves during geomagnetic storms, J. Geophys. Res., 108(A4), 1143, doi:10.1029/2002JA009489, 2003.

Summers, D., R. M. Thorne, and F. Xiaio, Relativistic theory of waveparticle resonant diffusion with application to electron acceleration in the magnetosphere, J. Geophys. Res., 103, 20,487, 1998.

Summers, D., C. Ma, N. P. Meredith, R. B. Horne, R. M. Thorne, D. Heynderickx, and R. R. Anderson, Model of the energization of outer-zone electrons by whistler mode chorus during the October 9, 1990 geomagnetic storm, Geophys. Res. Lett., 29(24), 2174, doi:10.1029/ 2002GL016039, 2002.

Thorne, R. M., A possible cause of dayside relativistic electron precipitation events, J. Atmos. Terr. Phys., 36, 635, 1974.

Thorne, R. M., and C. F. Kennel, Relativistic electron precipitation during magnetic storm main phase, J. Geophys. Res., 76, 4446, 1971.

Thorne, R. M., and L. J. Andreoli, Mechanisms for intense relativistic electron precipitation, in Exploration of the Polar Upper Atmosphere, edited by C. S. Deehr and J. A. Holtet, p. 381, D. Reidel, Norwell, Mass., 1980.

Thorne, R. M., and R. B. Horne, Modulation of electromagnetic ion cyclotron instability due to interaction with ring current $\mathrm{O}^{+}$during magnetic storms, J. Geophys. Res., 102, 14,155, 1997.

Vampola, A. L., The aerospace environment at high altitudes and its implications for spacecraft charging and communications, J. Electrostat, 20, 21, 1987.

Walker, A. D. M., Plasma Waves in the Magnetosphere, Springer-Verlag, New York, 1993.

Wrenn, G. L., Conclusive evidence for internal dielectric charging anomalies on geosynchronous communications spacecraft, J. Spacecr. Rockets, $32,514,1995$

Wrenn, G. L., and R. J. K. Smith, The ESD threat to GEO satellites: Empirical models for observed effects due to both surface and internal charging, in ESA Symposium Proceedings on Environment Modelling for Space-Based Applications, ESA SP-392, Eur. Space Agency, Noorwijk, Netherlands, 1996.

Young, D. T., Heavy ion plasmas in the outer magnetosphere, J. Geophys., $52,167,1983$

Young, D. T., J. Geiss, H. Balsiger, P. Eberhardt, A. Ghielmetti, and H. Rosenbauer, Discovery of $\mathrm{He}^{2+}$ and $\mathrm{O}^{2+}$ ions of terrestrial origin in the outer magnetosphere, Geophys. Res. Lett., 4, 561, 1977.

Young, D. T., S. Perraut, A. Roux, C. de Villedaro, R. Gendrin, A. Korth, G. Kremser, and D. Jones, Wave-particle interactions near $\Omega_{\mathrm{H}^{+}}$observed on GEOS 1 and 2: 1. Propagation of ion cyclotron waves in $\mathrm{He}^{+}-$rich plasma, J. Geophys. Res., 86, 6755, 1981.

R. R. Anderson, Department of Physics and Astronomy, The University of Iowa, Iowa City, Iowa, IA 52242-1479, USA. (roger-r-anderson@uiowa. edu)

B. J. Fraser, Cooperative Research Centre for Satellite Systems, Department of Physics, University of Newcastle, NSW 2308, Australia. (phbjf@cc.newcastle.edu.au)

R. B. Horne, British Antarctic Survey, Natural Environment Research Council, Madingley Road, Cambridge, CB3 0ET, UK. (r.horne@bas.ac.uk)

N. P. Meredith, Mullard Space Science Laboratory, University College London, Holmbury St. Mary, Dorking, Surrey, RH5 6NT, UK. (npm@mssl. ucl.ac.uk)

D. Summers, Department of Mathematics and Statistics, Memorial University of Newfoundland, St. John's, Newfoundland, Canada, A1C 5S7. (dsummers@math.mun.ca)

R. M. Thorne, Department of Atmospheric Sciences, University of California, Los Angeles, 405 Hilgard Avenue, Los Angeles, CA 90095 1565, USA. (rmt@atmos.ucla.edu) 